\title{
One-bipolar topologically slice knots and primary decomposition
}

\author{
Min Hoon Kim, Se-Goo Kim, and Taehee Kim
}

\begin{abstract}
Let $\left\{\mathcal{T}_{n}\right\}$ be the bipolar filtration of the smooth concordance group of topologically slice knots, which was introduced by Cochran, Harvey, and Horn. It is known that for each $n \neq 1$ the group $\mathcal{T}_{n} / \mathcal{T}_{n+1}$ has infinite rank and $\mathcal{T}_{1} / \mathcal{T}_{2}$ has positive rank. In this paper, we show that $\mathcal{T}_{1} / \mathcal{T}_{2}$ also has infinite rank. Moreover, we prove that there exist infinitely many Alexander polynomials $p(t)$ such that there exist infinitely many knots in $\mathcal{T}_{1}$ with Alexander polynomial $p(t)$ whose nontrivial linear combinations are not concordant to any knot with Alexander polynomial coprime to $p(t)$, even modulo $\mathcal{T}_{2}$. This extends the recent result of Cha on the primary decomposition of $\mathcal{T}_{n} / \mathcal{T}_{n+1}$ for all $n \geq 2$ to the case $n=1$.

To prove our theorem, we show that the surgery manifolds of satellite links of $\nu^{+}$-equivalent knots with the same pattern link have the same Ozsváth-Szabó $d$-invariants, which is of independent interest. As another application, for each $g \geq 1$, we give a topologically slice knot of concordance genus $g$ that is $\nu^{+}$-equivalent to the unknot.
\end{abstract}

\section{Introduction}

Since Freedman Fre82b, Fre84] and Donaldson [Don83] revealed the difference between the smooth and topological structures on 4-manifolds, classifying the structure of the concordance group of topologically slice knots, which we denote by $\mathcal{T}$, has been one of the central research subjects. Freedman showed that knots with trivial Alexander polynomial are topologically slice [Fre82a, FQ90], so $\mathcal{T}$ has a subgroup $\Delta$ generated by knots with trivial Alexander polynomial. By using gauge theory and Heegaard Floer homology, it is known that $\mathcal{T}$ and $\Delta$ have $\mathbb{Z}^{\infty}$-summands [End95, HK11, HK12, OSS17, DHSL19, KP18, The difference between $\mathcal{T}$ and $\Delta$ was found recently, and it is known that the group $\mathcal{T} / \Delta$ has a $\mathbb{Z}^{\infty} \oplus \mathbb{Z}_{2}^{\infty}$ subgroup [HLR12, HKL16, CH15].

Cochran, Harvey, and Horn [CHH13] established a filtration indexed by nonnegative integers

$$
0 \subset \cdots \subset \mathcal{T}_{n+1} \subset \mathcal{T}_{n} \subset \cdots \subset \mathcal{T}_{0} \subset \mathcal{T}
$$

which is called the bipolar filtration of $\mathcal{T}$, where $\mathcal{T}_{n}$ is the subgroup of (the concordance classes of) $n$-bipolar knots. We give a definition of $\mathcal{T}_{n}$ in Definition 2.1 Briefly, the notion of an $n$-bipolar knot is based on the idea of relating Donaldson's diagonalization theorem to the covering spaces of a 4-manifold with boundary the zero-framed surgery on the knot in $S^{3}$ corresponding to the derived series of the fundamental group of the 4-manifold. The bipolar filtration of $\mathcal{T}$ also descends to a filtration of $\mathscr{T}:=\mathcal{T} / \Delta$ :

$$
0 \subset \cdots \subset \mathscr{T}_{n+1} \subset \mathscr{T}_{n} \subset \cdots \subset \mathscr{T}_{1} \subset \mathscr{T}_{0} \subset \mathscr{T}
$$

where $\mathscr{T}_{n}:=\mathcal{T}_{n} /\left(\mathcal{T}_{n} \cap \Delta\right)$.

2010 Mathematics Subject Classification. 57N13, 57M27, 57N70, 57M25.

The first named author was partly supported by the POSCO TJ Park Science Fellowship. The second named author was supported by the Basic Science Research Program through the National Research Foundation of Korea (NRF) funded by the Ministry of Education (NRF-2015R1D1A1A01058384). The last named author was supported by Basic Science Research Program through the National Research Foundation of Korea (NRF) funded by the Ministry of Education (no.2018R1D1A1B07048361). 
Many concordance invariants derived from the Floer homology theory by Ozsváth and Szabó vanish on $\mathcal{T}_{0}$ or $\mathcal{T}_{1}$. In CHH13, it was shown that the $\tau$-invariant OS03b, $\varepsilon$-invariant Hom14, and $\delta_{p}$-invariant MO07, Jab12] vanish for knots in $\mathcal{T}_{0}$. It is known that the $\nu^{+}$-invariant [HW16, the $\Upsilon$-invariant OSS17, and the $\varphi_{j}$-invariants [DHSL19] vanish for knots in $\mathcal{T}_{0}$, and also the various invariants in JN07, GRS08, GJ11 derived from the correction term $d$-invariants of Ozsváth and Szabó OS03a vanish for knots in $\mathcal{T}_{1}$ (see [CHH13, Section 6]).

Nevertheless, combining the $d$-invariant with topological concordance invariants such as the von Neumann $\rho$-invariant and the Casson-Gordon invariant, it was found that the bipolar filtrations are highly nontrivial; it was shown that $\mathcal{T} / \mathcal{T}_{0}$ has infinite rank by Cochran, Harvey, and Horn CHH13. It was also shown that $\mathcal{T}_{n} / \mathcal{T}_{n+1}$ has infinite rank for $n=0$ by Cochran and Horn [CH15] and for $n \geq 2$ by Cha and the first named author CK17. For the filtration $\left\{\mathscr{T}_{n}\right\}$ of $\mathscr{T}$, Cochran and Horn CH15] showed that $\mathscr{T}_{0} / \mathscr{T}_{1}$ has infinite rank, and Cha Cha19] showed that $\mathscr{T}_{n} / \mathscr{T}_{n+1}$ has infinite rank for each $n \geq 2$.

But for the remaining case $n=1$, it is only known that $\mathcal{T}_{1} / \mathcal{T}_{2}$ has positive rank CHH13 and it remains as an open question whether or not $\mathcal{T}_{1} / \mathcal{T}_{2}$ and $\mathscr{T}_{1} / \mathscr{T}_{2}$ have infinite rank. The first main result of this paper is to answer the question:

Theorem 1.1. The groups $\mathcal{T}_{1} / \mathcal{T}_{2}$ and $\mathscr{T}_{1} / \mathscr{T}_{2}$ have infinite rank.

Very recently, Cha Cha19 presented a framework for the study on the primary decomposition of $\mathcal{T}$. Theorem 1.1 immediately follows from Theorem 1.2 below which reveals a new structure of the primary decomposition of $\mathcal{T}_{1} / \mathcal{T}_{2}$ and $\mathscr{T}_{1} / \mathscr{T}_{2}$ by extending Theorems C and D in Cha19 for $n=1$.

To state our result more explicitly, we set up notations.

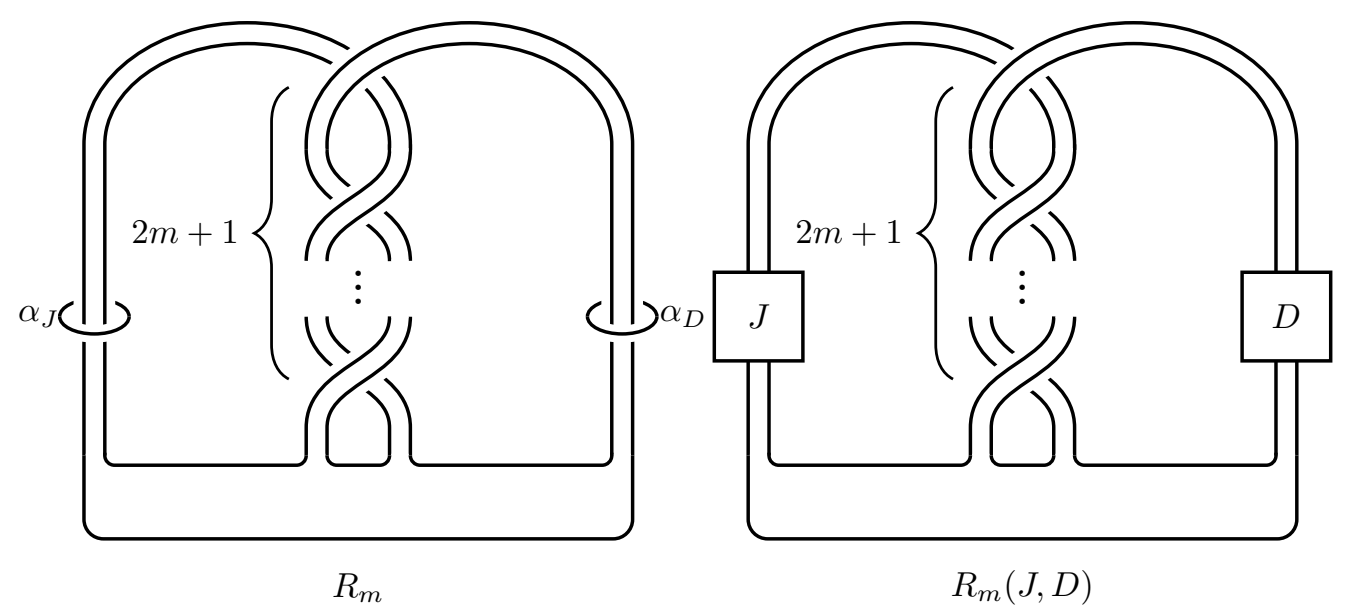

FiguRE 1. The knot $R_{m}$ and the satellite knot $R_{m}(J, D)$.

Let $R_{m}$ be the genus one knot with Alexander polynomial $\lambda_{m}=(m t-(m+1))((m+$ 1) $t-m) \in \mathbb{Z}\left[t^{ \pm 1}\right]$ and $\alpha_{J}$ and $\alpha_{D}$ be the curves dual to the bands of the Seifert surface of $R_{m}$ as depicted in Figure 1 . For knots $J$ and $D$, we denote by $R_{m}(J, D)$ the knot obtained via the satellite construction which ties the knot $J$ (resp. $D$ ) through the band dual to the curve $\alpha_{J}$ (resp. $\alpha_{D}$ ) as depicted in Figure 1. For a knot $K$ and $t \in \mathbb{Z}$, let $D_{+}(K, t)$ (resp. $\left.D_{-}(K, t)\right)$ denote the $t$-twisted positive-clasped (resp. negative-clasped) Whitehead double of $K$. Let $D:=D_{+}\left(T_{2,3}, 0\right)$ for the right-handed trefoil $T_{2,3}$. For 
$i \geq 1$, let $p_{i}$ be primes such that $5=p_{1}<p_{2}<\cdots$ and each $p_{i}$ is congruent to 1 modulo 4. Let $k_{i}:=\frac{1}{8}\left(p_{i}-1\right)^{2}$ and $J_{i}:=D_{-}\left(k_{i} T_{2,3}, 2 k_{i}\right)$.

For a knot $K$ let $\Delta_{K}$ denote the Alexander polynomial of $K$. Let

$$
\mathcal{T}^{\lambda_{m}}:=\left\{[K] \in \mathcal{C} \mid \Delta_{K} \text { is coprime to } \lambda_{m}\right\},
$$

and for a subgroup $S$ of $\mathcal{C}$ let $S^{\lambda_{m}}:=S \cap \mathcal{T}^{\lambda_{m}}, \mathscr{S}:=S / S \cap \Delta$, and $\mathscr{S}^{\lambda_{m}}:=S^{\lambda_{m}} / S^{\lambda_{m}} \cap \Delta$. Note that if $S$ is a subgroup of $\mathcal{T}, \mathscr{S}$ is a subgroup of $\mathscr{T}$. The groups $\mathcal{T}_{1} /\left(\mathcal{T}_{1}^{\lambda_{m}}+\mathcal{T}_{2}\right)$ and $\mathscr{T}_{1} /\left(\mathscr{T}_{1}^{\lambda_{m}}+\mathscr{T}_{2}\right)$ are isomorphic since $\mathcal{T}_{1} \cap \Delta$ is contained in $\mathcal{T}_{1}^{\lambda_{m}}$.

Theorem 1.2. Let $m$ be an odd positive integer. For all $i \geq 1$, the knots $R_{m}\left(J_{i}, D\right)$, which have Alexander polynomial $\lambda_{m}$, are in $\mathcal{T}_{1}$. If $K$ is a nontrivial linear combination of $R_{m}\left(J_{i}, D\right)$, then $K$ is not concordant to any knot with Alexander polynomial coprime to $\lambda_{m}$ modulo $\mathcal{T}_{2}$. In particular, the groups $\mathcal{T}_{1} /\left(\mathcal{T}_{1}^{\lambda_{m}}+\mathcal{T}_{2}\right)$ and $\mathscr{T}_{1} /\left(\mathscr{T}_{1}^{\lambda_{m}}+\mathscr{T}_{2}\right)$ have infinite rank.

Since $\mathcal{T}_{1} / \mathcal{T}_{2}$ and $\mathscr{T}_{1} / \mathscr{T}_{2}$ surject to $\mathcal{T}_{1} /\left(\mathcal{T}_{1}^{\lambda_{m}}+\mathcal{T}_{2}\right)$ and $\mathscr{T}_{1} /\left(\mathscr{T}_{1}^{\lambda_{m}}+\mathscr{T}_{2}\right)$, respectively, Theorem 1.1 follows immediately from Theorem 1.2 . We remark that Theorem $1.2 \mathrm{ex}-$ tends Cha19, Theorems $\mathrm{C}$ and D] which show that the groups $\mathcal{T}_{n} /\left(\mathcal{T}_{n}^{\lambda_{m}}+\mathcal{T}_{n+1}\right)$ and $\mathscr{T}_{n} /\left(\mathscr{T}_{n}^{\lambda_{m}}+\mathscr{T}_{n+1}\right)$ have infinite rank for $n \geq 2$.

To prove Theorem 1.2 we use the arguments in CHH13, CK17, Cha19, which combine the $d$-invariant and the von Neumann $\rho$-invariant, and in particular estimate the $d$ invariants of branched covers of $S^{3}$ along $R_{m}(J, D)$ for some knots $J$ (see [CHH13, Lemma 8.2] and [CK17, Lemma 5.1]). To be precise, in [CHH13, Lemma 8.2] and [CK17, Lemma 5.1], the authors show that certain $d$-invariants of branched covers of $S^{3}$ along $R_{m}(U, D)$ are negative where $U$ is the unknot, and the knots $J$ are needed to be unknotted by changing only positive crossings to conclude that the corresponding $d$-invariants of branched covers of $S^{3}$ along $R_{m}(J, D)$ are also negative. Due to this restriction on $J$, one could not apply directly the arguments in CHH13, CK17, Cha19 to the case of $\mathcal{T}_{1} / \mathcal{T}_{2}$ and show $\mathcal{T}_{1} / \mathcal{T}_{2}$ has infinite rank; note that our knots $J_{i}$ in Theorem 1.2 are not unknotted by changing only positive crossings.

To resolve this issue and prove Theorem 1.2 , we show that the surgery manifolds of satellite links of $\nu^{+}$-equivalent knots with the same pattern link have the same $d$ invariants, which is of independent interest. (For the definition of $\nu^{+}$-equivalence, see Definition 2.2.) In the following, $P(K)$ and $P(J)$ denote the satellite links of knots $K$ and $J$ with pattern link $P$, respectively.

Theorem 1.3. Let $K$ and $J$ be knots and $P$ be an ordered $k$-component link in $S^{1} \times D^{2}$. Let $\Lambda=\left(n_{1}, \ldots, n_{k}\right)$ be surgery coefficients such that $S_{\Lambda}^{3}(P(K))$ and $S_{\Lambda}^{3}(P(J))$ are $\mathbb{Z}_{2}$ homology spheres. If $K$ and $J$ are $\nu^{+}$-equivalent, then

$$
d\left(S_{\Lambda}^{3}(P(K)), \mathfrak{t}_{K}\right)=d\left(S_{\Lambda}^{3}(P(J)), \mathfrak{t}_{J}\right)
$$

where $\mathfrak{t}_{K}$ and $\mathfrak{t}_{J}$ are $\operatorname{Spin}^{c}$ structures satisfying $c_{1}\left(\mathfrak{t}_{K}\right)=c_{1}\left(\mathfrak{t}_{J}\right)$ under the bijection $H^{2}\left(S_{\Lambda}^{3}(P(K))\right) \rightarrow H^{2}\left(S_{\Lambda}^{3}(P(J))\right)$.

Theorem 1.3 is proved in Subsection 2.3 . This can be regarded as a link analogue of the fact that satellite knots of $\nu^{+}$-equivalent knots with the same pattern knot are $\nu^{+}$-equivalent which was proved in KP18, Sat18.

Theorem 1.2 has an application to concordance genus. Recall that the concordance genus of a knot is the minimum genus among all knots concordant to the knot. Using the $\varepsilon$-invariant Hom Hom15. showed that for each $g \geq 1$ there exists a topologically slice knot of concordance genus $g$ and 4-genus one. There are also lower bounds on concordance genus obtained from the $\Upsilon$-invariant OSS17] and the $\varphi_{j}$-invariants [DHSL19. Note that if a knot is $\nu^{+}$-equivalent to the unknot, then it has vanishing $\varepsilon$-invariant and $\Upsilon$-invariant Hom17, and it also has vanishing $\varphi_{j}$-invariants DHSL19. 
Theorem 1.4. For each $g \geq 1$, there exists a topologically slice knot of concordance genus $g$ which is $\nu^{+}$-equivalent to the unknot.

Proof. For $1 \leq i \leq g$, let $K_{i}:=R_{2 i-1}\left(J_{1}, D\right)$ and $K:=\#_{i=1}^{g} K_{i}$ (recall that $J_{1}=$ $D_{-}\left(2 T_{2,3}, 4\right)$ and $\left.D=D_{+}\left(T_{2,3}, 0\right)\right)$. Then, $K$ is a topologically slice knot of genus $g$ and the degree of $\Delta_{K}$ is $2 g$.

Since $K$ is in $\mathcal{T}_{1}$, by CHH13, Corollary 6.7] $d\left(S_{1}^{3}(K)\right)=0$. Therefore $V_{0}(K)=$ $-\frac{1}{2} d\left(S_{1}^{3}(K)\right)=0$ where $V_{0}$ is a knot concordance invariant given in Subsection 2.2 of NW15. Since $\nu^{+}$is the smallest $k \geq 0$ such that $V_{k}=0$, it follows that $\nu^{+}(K)=0$ and hence $K$ is $\nu^{+}$-equivalent to the unknot.

Suppose $J$ is a knot of genus $g^{\prime}<g$ and $K \#-J$ is slice. Since the degree of $\Delta_{J}$ is less than $2 g$, there is some $j \in\{1,2, \ldots, g\}$ such that $\Delta_{K_{j}}$ is not a factor of $\Delta_{J}$. Then, for $K^{\prime}:=\left(\left(\# i \neq j K_{i}\right) \#-J\right)$, we have $K_{j} \# K^{\prime}=K \#-J$ is slice and $\Delta_{K_{j}}$ is coprime to $\Delta_{K^{\prime}}$. This contradicts Theorem 1.2

We remark that Theorem 1.4 can also be shown using the knots in Cha19, Theorem D].

If a topologically slice knot $K$ is not in $\mathcal{T}_{1}$, one cannot show that $K$ is nontrivial in $\mathcal{T} / \mathcal{T}^{\Delta_{K}}$ using the arguments in the proof of Theorem 1.2. In Appendix, combining the results in Kim05, KK08, Bao15, KK18, we give an obstruction for a knot $K$ to being in $\mathcal{T}^{\Delta_{K}}$ (see Theorem A.1), and using it we show that the knot $R_{1}(J, D)$ with $J:=D_{+}\left(T_{2,3}, 0\right) \#-T_{2,3}$ is nontrivial in $\mathcal{T} / \mathcal{T}^{\lambda_{1}}$ (see Proposition A.2. It is unknown to the authors whether or not the knot $R_{1}(J, D)$ is in $\mathcal{T}_{1}$.

The rest of the paper is organized as follows. In Section 2, we introduce the bipolar filtration and prove Theorem 1.3, and in Section 3 we prove Theorem 1.2. In Appendix, we prove Theorem A.1.

In this paper, all homology groups are understood with integer coefficients unless mentioned otherwise.

Acknowledgements. The authors thank Jae Choon Cha for sharing with them his insight on the subject and his early drafts of Cha19.

\section{The bipolar filtration, satellite links and $\nu^{+}$-equivalence}

\subsection{The bipolar filtration}

In this subsection, we recall the definition of the bipolar filtration $\left\{\mathcal{T}_{n}\right\}$ introduced in CHH13. Throughout this paper, for a knot $K$ we denote by $M(K)$ the zero-framed surgery on $K$ in $S^{3}$. For a group $G$, we define $G^{(0)}:=G$ and $G^{(n+1)}:=\left[G^{(n)}, G^{(n)}\right]$ for each $n \geq 0$.

Definition 2.1 ([CHH13, Definition 5.1]). Let $n \geq 0$ be an integer. A knot $K$ is $n$ positive if $M(K)$ bounds a compact connected oriented smooth 4-manifold $W$ which satisfies the following.

(1) The inclusion-induced homomorphism $H_{1}(M(K)) \rightarrow H_{1}(W)$ is an isomorphism and $\pi_{1}(W)$ is normally generated by a meridian of $K$.

(2) $H_{2}(W)$ has a basis which consists of disjointly embedded compact connected oriented surfaces $S_{i}, 1 \leq i \leq r$, such that for each $i$, the surface $S_{i}$ has a normal bundle of Euler class 1 and $\pi_{1}\left(S_{i}\right) \subset \pi_{1}(W)^{(n)}$.

The 4-manifold $W$ is called an $n$-positon. Similarly, by changing the Euler class condition from 1 to -1, we define an n-negative knot and an n-negaton. A knot is n-bipolar if it is $n$-positive and $n$-negative. We let $\mathcal{T}_{n}:=\{[K] \in \mathcal{T} \mid K$ is $n$-bipolar $\}$. 
Each $\mathcal{T}_{n}$ is a subgroup of $\mathcal{T}$ and since for $m \geq n$, an $m$-bipolar knot is $n$-bipolar, we have a descending filtration

$$
0 \subset \cdots \subset \mathcal{T}_{n+1} \subset \mathcal{T}_{n} \subset \cdots \subset \mathcal{T}_{1} \subset \mathcal{T}_{0} \subset \mathcal{T} .
$$

\section{2. $\nu^{+}$-equivalence}

Let $\nu^{+}$be the knot concordance invariant introduced by Hom and Wu HW16]. Using the $\nu^{+}$-invariant, one can consider the following equivalence relation coarser than concordance.

Definition 2.2. Two knots $K$ and $J$ in $S^{3}$ are $\nu^{+}$-equivalent if

$$
\nu^{+}(K \#-J)=\nu^{+}(-K \# J)=0 .
$$

Remark 2.3. By [Hom17, Proposition 3.11], the following conditions are equivalent (see also [KP18, Lemma 3.1]).

(1) Two knots $K$ and $J$ are $\nu^{+}$-equivalent.

(2) $C F K^{\infty}(K)$ and $C F K^{\infty}(J)$ are stably chain homotopy equivalent. More precisely, there are two acyclic chain complexes $A$ and $A^{\prime}$ such that $C F K^{\infty}(K) \oplus A$ and $C F K^{\infty}(J) \oplus A^{\prime}$ are filtered chain homotopy equivalent.

(3) $d_{ \pm 1 / 2}\left(S_{0}^{3}(K \#-J)\right)=d_{ \pm 1 / 2}\left(S_{0}^{3}(J \#-K)\right)= \pm \frac{1}{2}$.

For example, $D_{+}\left(T_{2,3}\right) \#-T_{2,3}$ is $\nu^{+}$-equivalent to the unknot since $C F K^{\infty}\left(D_{+}\left(T_{2,3}\right)\right)$ is filtered chain homotopy equivalent to $C F K^{\infty}\left(T_{2,3}\right) \oplus A$ for some acyclic chain complex $A$ by Proposition 6.1 of [HKL16.

\subsection{Satellite links with $\nu^{+}$-equivalent companions}

We recall Theorem 1.3 below.

Theorem 2.4. Let $K$ and $J$ be knots and $P$ be an ordered $k$-component link in $S^{1} \times D^{2}$. Let $\Lambda=\left(n_{1}, \ldots, n_{k}\right)$ be surgery coefficients such that $S_{\Lambda}^{3}(P(K))$ and $S_{\Lambda}^{3}(P(J))$ are $\mathbb{Z}_{2}$ homology spheres. If $K$ and $J$ are $\nu^{+}$-equivalent, then

$$
d\left(S_{\Lambda}^{3}(P(K)), \mathfrak{t}_{K}\right)=d\left(S_{\Lambda}^{3}(P(J)), \mathfrak{t}_{J}\right)
$$

where $\mathfrak{t}_{K}$ and $\mathfrak{t}_{J}$ are $\operatorname{Spin}^{c}$ structures satisfying $c_{1}\left(\mathfrak{t}_{K}\right)=c_{1}\left(\mathfrak{t}_{J}\right)$ under the canonical bijection $H^{2}\left(S_{\Lambda}^{3}(P(K))\right) \rightarrow H^{2}\left(S_{\Lambda}^{3}(P(J))\right)$.

Proof. We prove Theorem 2.4 assuming Proposition 2.5 below. Let $a \in G$ be the image of $c_{1}\left(\mathfrak{t}_{K}\right)$ via the isomorphism given in Proposition 2.5 (1). By Proposition 2.5 (3), $(0, a) \in \mathbb{Z} \oplus G \cong H^{2}(Z)$ is a characteristic element, so we can choose a $\operatorname{Spin}^{c}$ structure $\mathfrak{s}$ on $Z$ such that $\mathfrak{s}$ restricts to $\mathfrak{t}_{0}, \mathfrak{t}_{K}$ and $\mathfrak{t}_{J}$ on $S_{0}^{3}(J \#-K), S_{\Lambda}^{3}(P(K))$, and $S_{\Lambda}^{3}(P(J))$, respectively, where $\mathfrak{t}_{0}$ is the torsion $\operatorname{Spin}^{c}$ structure on $S_{0}^{3}(J \#-K)$. Since $b_{1}(\partial Z)=1$, $\partial Z$ has trivial triple cup product, and hence $\partial Z$ has standard $H F^{\infty}$ by Theorem 3.2 of [LR14. Since $Z$ is negative semidefinite, by Theorem 4.7 of [LR14],

$$
0 \leq 4 d_{b o t}\left(\partial Z,\left.\mathfrak{s}\right|_{\partial Z}\right)+2 .
$$

On the other hand, $\left(\partial Z,\left.\mathfrak{s}\right|_{\partial Z}\right)=\left(S_{\Lambda}^{3}(P(K)), \mathfrak{t}_{K}\right) \#\left(-S_{\Lambda}^{3}(P(J)), \mathfrak{t}_{J}\right) \#\left(S_{0}^{3}(J \#-K), \mathfrak{t}_{0}\right)$. By the additivity in [LR14, Proposition 4.3], the inequality becomes

$$
0 \leq 4 d\left(S_{\Lambda}^{3}(P(K)), \mathfrak{t}_{K}\right)-4 d\left(S_{\Lambda}^{3}(P(J)), \mathfrak{t}_{J}\right)+4 d_{-1 / 2}\left(S_{0}^{3}(J \#-K)\right)+2 .
$$

Since $K$ and $J$ are $\nu^{+}$-equivalent, by Remark 2.3 . $d_{-1 / 2}\left(S_{0}^{3}(J \#-K)\right)=-\frac{1}{2}$. It follows that

$$
d\left(S_{\Lambda}^{3}(P(K)), \mathfrak{t}_{K}\right) \geq d\left(S_{\Lambda}^{3}(P(J)), \mathfrak{t}_{J}\right) .
$$

By changing the roles of $J$ and $K$, we obtain the desired equality. 
Proposition 2.5. Under the same hypothesis as in Theorem 2.4, there is a compact connected oriented smooth 4 -manifold $Z$ with $\partial Z \cong S_{\Lambda}^{3}(P(K)) \#-S_{\Lambda}^{3}(P(J)) \# S_{0}^{3}(J \#-K)$ satisfying the following.

(1) $H^{2}(Z) \cong \mathbb{Z} \oplus G$ such that the $\mathbb{Z}$-summand is isomorphic to $H^{2}\left(S_{0}^{3}(J \#-K)\right)$ and the group $G$ is isomorphic to both $H^{2}\left(S_{\Lambda}^{3}(P(K))\right)$ and $H^{2}\left(S_{\Lambda}^{3}(P(J))\right)$ via the inclusion-induced maps. The composition of the isomorphisms

$$
H^{2}\left(S_{\Lambda}^{3}(P(K))\right) \longrightarrow G \longrightarrow H^{2}\left(S_{\Lambda}^{3}(P(J))\right)
$$

coincide with the canonical bijection $H^{2}\left(S_{\Lambda}^{3}(P(K))\right) \rightarrow H^{2}\left(S_{\Lambda}^{3}(P(J))\right)$.

(2) $H_{1}(Z) \cong G$.

(3) The intersection form of $Z$ is trivial. In particular, $Z$ is negative semidefinite.

For an ordered, oriented link $P$ in $S^{1} \times D^{2}$, let $-P$ be the link in $S^{1} \times D^{2}$ which is the mirror of $P$ with the reversed string orientations. Denote by $H$ the genus 2 handlebody. Regard $H$ as the boundary connected sum of two copies of $S^{1} \times D^{2}$. Consider two links $P \sqcup-P$ and $C$ in $H$ in Figure 2, We need a lemma.
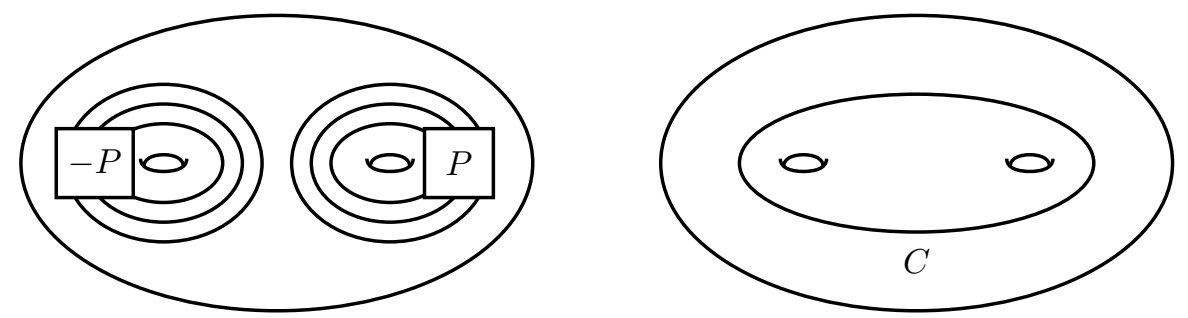

Figure 2. The links $P \sqcup-P$ and $C$ in $H$.

Lemma 2.6. For each ordered, oriented $k$-component link $P$ in $S^{1} \times D^{2}$, there exist compact connected surfaces $S_{1}, \ldots, S_{k}$ of genus 0 with $\partial S_{i} \subset H \times\{0,1\}$ which are properly disjointly embedded in $H \times[0,1]$ such that the following hold:

(1) For each $i, \partial S_{i} \cap H \times\{0\}$ is either the empty set or the union of finitely many parallel copies of $C$ endowed with a certain orientation.

(2) For each $i, \partial S_{i} \cap H \times\{1\}$ is the union of the $i$-th components of $P$ and $-P$. In particular, $\left(\bigsqcup_{i=1}^{k} \partial S_{i}\right) \cap H \times\{1\}=P \sqcup-P$.

Before proving this lemma, we define a couple of terms: a diagram of a link $L$ in $H$ is the projection of $L$ into the boundary connected sum of two annuli, a twice punctured disk, having transversal double singularities equipped with under and over passing information. Here, $H$ is assumed to be embedded in $\mathbb{R}^{3}$ in a standard way, whose projection is a twice punctured disk. The singularities of the diagram are called crossings.

Proof of Lemma 2.6. Let $P_{i}$ be the $i$-th component of $P$ for $i=1, \ldots, k$. Let $M$ be the disk, where the boundary connected sum of $H=S^{1} \times D^{2} \natural S^{1} \times D^{2}$ takes place. One may consider $M$ as the mirror reflecting $P$ onto $-P$ in $H$. In other words, there is an involution $\iota$ on $H$ fixing each point of $M$ and $\iota\left(P_{i}\right)=-P_{i}$ for $i=1, \ldots, k$.

For each $i=1, \ldots, k$, we can choose a simple path $\gamma_{i}$ from a point of $P_{i}$ to a point of $-P_{i}$ so that $\iota\left(\gamma_{i}\right)=\gamma_{i}, \gamma_{i}$ meets each of $M, P_{i}$, and $-P_{i}$ in exactly one point, but $\gamma_{i}$ does not meet $P_{j}$ or $\gamma_{j}$ for any $j \neq i$. We take band sums of $P_{i}$ with $-P_{i}$ along $\gamma_{i}$ for all $i$. This procedure gives us a link $L$ in $H$ satisfying the following:

(i) Each component is of the form $K \# \iota(K)$ for some knot $K$ in $S^{1} \times D^{2}$.

(ii) Each component intersects the mirror $M$ in exactly two points. 
It also produces properly, disjointly embedded, compact connected surfaces $S_{1}^{\prime}, \ldots, S_{k}^{\prime}$ of genus 0 in $S^{3} \times\left[\frac{1}{2}, 1\right]$ such that $\partial S_{i}^{\prime} \cap S^{3} \times\{1\}=P_{i} \sqcup-P_{i}$ and $\partial S_{i}^{\prime} \cap S^{3} \times\left\{\frac{1}{2}\right\}=P_{i} \#-P_{i}$. Note that, if a link $L$ in $H$ satisfies conditions (i) and (ii), then, for every crossing $c$ of $L$, the overpassing arcs of $c$ and $\iota(c)$ belong to the same component of $L$ and the underpassing arcs of $c$ and $\iota(c)$ belong to the same component of $L$.

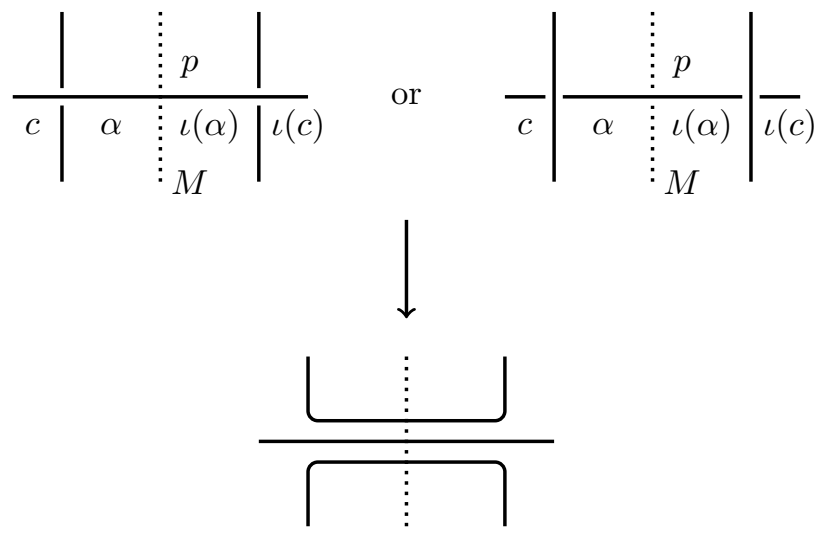

FiguRE 3. Band move.

Beginning with a diagram $D$ of such a link $L$, we shall find a sequence of band sums, each of which decreases the number of crossings and increases the number of components. So, the final link consists of unlinked unknots and unlinked parallel copies of $C$. The traces of the band sums and the disks bounding the unlinked unknots give disjointly embedded, compact connected surfaces $S_{1}^{\prime \prime}, \ldots, S_{k}^{\prime \prime}$ of genus 0 in $S^{3} \times\left[0, \frac{1}{2}\right]$ such that $\partial S_{i}^{\prime \prime} \cap S^{3} \times\left\{\frac{1}{2}\right\}=P_{i} \#-P_{i}$ and $\partial S_{i}^{\prime \prime} \cap S^{3} \times\{0\}$ is either the empty set or the union of finitely many parallel copies of $C$. By taking $S_{i}$ to be the union of $S_{i}^{\prime}$ and $S_{i}^{\prime \prime}$, this will prove the lemma.

We use induction on the number of crossings in $D$. Suppose $D$ has no crossing. Then, each component is either parallel to $C$ or unknotted in $H$. Use the innermost circle argument to cap off the unknotted components, and we are done for this case. Now assume that $D$ has a crossing. Let $K$ be a knot in $S^{1} \times D^{2}$ for which $K \# \iota(K)$ is a component of $D$ having a crossing. As stated above, the component meets $M$ at a point $p$. Let $c$ be the crossing in $K$ from which to $p$ the part of $K$ has no crossings. Denote the part by $\alpha$. Then $\alpha \cup \iota(\alpha)$ is a path from $c$ to $\iota(c)$, which is a part of $K$ with no crossings. See Figure 3. Along this path $\alpha \cup \iota(\alpha)$, we take a band move between the two arcs that transversally cross $\alpha \cup \iota(\alpha)$ at $c$ and $\iota(c)$. It is obvious that this band sum reduces the number of crossings and increases the number of components. The resulting link has two less crossings and still satisfies the above two conditions (i) and (ii). Now, inductively we can find the desired sequence as we claimed above.

We are ready to prove Proposition 2.5 .

Proof of Proposition 2.5. For given two knots $K$ and $J$ in $S^{3}$, Figure 4 describes an embedding of $H \rightarrow S^{3}$. Let $f: H \times[0,1] \rightarrow S^{3} \times[0,1]$ denote the product of the embedding $H \rightarrow S^{3}$ and the identity on $[0,1]$. For a given $k$-component link $P$ in $S^{1} \times D^{2}$, by Lemma 2.6 , there are connected, disjointly embedded surfaces $S_{1}, \ldots, S_{k}$ of genus 0 in $H \times[0,1]$ such that $\partial S_{i} \cap H \times\{1\}$ is the union of the $i$-th component of $P$ and $-P$ for all $i$, and $\partial S_{i} \cap H \times\{0\}$ is either empty or the union of finitely many parallel copies of $C$ endowed with a certain orientation. By the definition of $f, f\left(S_{1}\right), \ldots, f\left(S_{k}\right)$ 
are disjointly embedded surfaces of genus 0 in $S^{3} \times[0,1]$ such that $\left(\bigsqcup_{i=1}^{k} f\left(S_{i}\right)\right) \cap S^{3} \times\{1\}$ is the split union $(P(K) \sqcup-P(J)) \times\{1\}$ and $\left(\bigsqcup_{i=1}^{k} f\left(S_{i}\right)\right) \cap S^{3} \times\{0\}$ is the union of finitely many parallel copies of $K \#-J$.

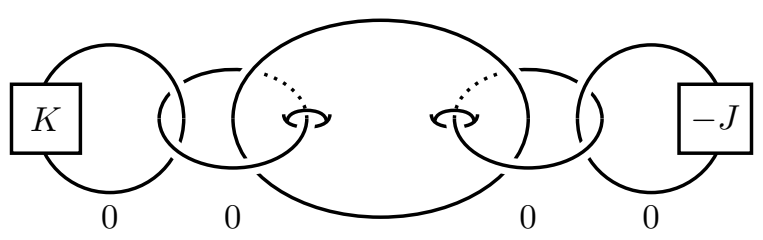

Figure 4. An embedding of $H$ in $S^{3}$.

Let $W$ be the 4-manifold obtained from $S^{3} \times[0,1]$ by attaching a 0 -framed 2-handle along $(K \#-J) \times\{0\}$. Note that $P(K) \times\{1\}$ and $-P(J) \times\{1\}$ cobound disjointly embedded annuli $A_{1}, \ldots, A_{k}$ in $W$. (To obtain $A_{i}$, cap off $f\left(S_{i}\right)$ by adding finitely many parallel copies of the 2 -handle core disk.) Let $Z_{0}$ be the result of $\Lambda=\left(n_{1}, \ldots, n_{k}\right)$ surgery on $W$ along the annuli $A_{1}, \ldots, A_{k}$. Note that $Z_{0}$ has two (oriented) boundary components $S_{\Lambda}^{3}(P(K)) \#-S_{\Lambda}^{3}(P(J))$ and $S_{0}^{3}(J \#-K)$. The desired 4-manifold $Z$ is obtained from $Z_{0}$ by deleting a neighborhood of an arc joining the boundary components of $Z_{0}$. Homologically, $Z$ is obtained from $D^{2} \times S^{2}$ by doing $\Lambda=\left(n_{1}, \ldots, n_{k}\right)$-surgeries on $k$ annuli, so the desired properties of $Z$ follow.

By the Akbulut-Kirby method AK80, we obtain surgery diagrams for the $r$-fold cyclic branched covers $\Sigma_{r}\left(R_{m}(J, D)\right)$ and $\Sigma_{r}\left(R_{m}(U, D)\right)$ of $S^{3}$ along $R_{m}(J, D)$ and $R_{m}(U, D)$ from the genus 1 Seifert surface in Figure 1. The surgery link of $\Sigma_{r}\left(R_{m}(J, D)\right)$ is obtained from that of $\Sigma_{r}\left(R_{m}(U, D)\right)$ by doing iterated satellite operations (actually $r$ times) with the fixed companion knot $J$. As in Theorem 2.4, we can identify the Spin $^{c}$ structures of $\Sigma_{r}\left(R_{m}(J, D)\right)$ and those of $\Sigma_{r}\left(R_{m}(U, D)\right)$. We remark that $\Sigma_{r}\left(R_{m}(J, D)\right)$ and $\Sigma_{r}\left(R_{m}(U, D)\right)$ are $\mathbb{Z}_{2}$-homology 3 -spheres since

$$
H_{1}\left(\Sigma_{r}\left(R_{m}(J, D)\right)\right) \cong H_{1}\left(\Sigma_{r}\left(R_{m}(U, D)\right)\right) \cong \mathbb{Z}_{N} \oplus \mathbb{Z}_{N}
$$

where $N=(m+1)^{r}-m^{r}$ (see [GL92, Proposition 2]).

Theorem 2.7. If $J$ is $\nu^{+}$-equivalent to the unknot $U$, then

$$
d\left(\Sigma_{r}\left(R_{m}(J, D)\right), \mathfrak{s}\right)=d\left(\Sigma_{r}\left(R_{m}(U, D)\right), \mathfrak{s}\right) .
$$

Proof. From the discussion given in the paragraph preceding Theorem 2.7, we obtain the conclusion by applying Theorem $2.4 n$ times.

\section{Proof of Theorem 1.2}

In this section we prove Theorem 1.2 The proof of Theorem 1.2 is essentially identical with the proof of [Cha19, Theorem D] with some modifications. Below we give a list of the key modifications for applying the proof of [Cha19. Theorem D] to the case $n=1$, which gives the proof of Theorem 1.2

(1) Use different $J_{i}$, as defined in the introduction of this paper, for the construction of $R_{m}\left(J_{i}, D\right)$.

(2) For the computation of the von Neumann $\rho$-invariants in Cha19, Section 3], in Cha19, Definition 3.1] take $n=1$ and replace the coefficients $\mathbb{Z}_{p_{1}}$ by $\mathbb{Q}$. This is due to our choice of $J_{i}$. 
(3) For the computation of correction terms $d\left(\Sigma_{r}\left(R_{m}\left(J_{i}, D\right)\right), \mathfrak{s}\right)$ in Cha19, Section 4], use Theorem 2.7 in place of [CHH13, Lemma 8.2] and [CK17, Lemma 5.1], which is again due to our choice of $J_{i}$. This is our key technical contribution for the proof of Theorem 1.2 .

The above key modifications with some minor changes for the proof of Theorem D in Cha19] will easily produce the proof of Theorem 1.2 .

Nonetheless, to clarify the proof, we give a more detailed sketch of the proof of Theorem 1.2 below following the arguments in the proof of Theorem D in [Cha19. In particular, we do not include some arguments in the proof of [Cha19, Theorem D] which are not necessary for our proof but were needed for the proof of [Cha19, Theorem D] to take care of far more general cases $n>1$.

For the rest of Section 3, we give a sketch of the proof of Theorem 1.2 Fix an odd positive integer $m$ and let $R:=R_{m}$. For each $i \geq 1$ let $K_{i}:=R\left(J_{i}, D\right)$ where $J_{i}=D_{-}\left(k_{i} T_{2,3}, 2 k_{i}\right)$ as defined in the introduction.

The following lemma gives the key properties of $J_{i}$. For a knot $K$, we denote by $\sigma_{K}$ the Levine-Tristram signature function of $K$ defined on $S^{1} \subset \mathbb{C}$.

Lemma 3.1. The knots $J_{i}$ satisfy the following.

(1) Each $J_{i}$ is 0-negative.

(2) For $i \geq 1, \int_{S^{1}} \sigma_{J_{i}}(\omega) d \omega$ are linearly independent over $\mathbb{Q}$.

(3) Each $J_{i}$ is $\nu^{+}$-equivalent to the unknot.

Proof. Since $J_{i}$ can be unknotted by changing a negative crossing, Property (1) follows from [CHH13, Proposition 3.1]. Since $\Delta_{J_{i}}=2 k_{i} t-\left(4 k_{i}-1\right)+2 k_{i} t^{-1}$, Property (2) follows from the proof of Proposition 2.6 in [COT04, p. 121]. For the concordance invariant $\tau$ of Ozsváth and Szabó [OS03b], Sato [Sat19, Theorem 1.2] showed that every genus 1 knot with $\tau=0$ is $\nu^{+}$-equivalent to the unknot. So, it is enough to show $\tau\left(J_{i}\right)=0$ to see Property (3). Note that $-J_{i}=D_{+}\left(k_{i} T_{2,-3},-2 k_{i}\right)$. Since $\tau\left(k_{i} T_{2,-3}\right)=-2 k_{i}$, we have $\tau\left(-J_{i}\right)=0$ by [Hed07, Theorem 1.5], and hence $\tau\left(J_{i}\right)=-\tau\left(-J_{i}\right)=0$.

Our knots $K_{i}$ are topologically slice and 1-bipolar, that is, $K_{i} \in \mathcal{T}_{1}$ for all $i \geq 1$; since $J_{i}$ is 0-negative (and $D$ is 0-positive), this follows from [CK17, Lemma 2.3].

Finally, we show that if $K$ is a nontrivial linear combination of $K_{i}$, then $K$ is not concordant to any knot with Alexander polynomial coprime to $\lambda_{m}$, modulo $\mathcal{T}_{2}$. Let $L$ be a knot with Alexander polynomial coprime to $\lambda_{m}$. For integers $a_{i}$, let $K:=\left(\#_{i=1}^{r} a_{i} K_{i}\right) \# L$. By renumbering indices and changing the orientation if necessary, we may assume that each $a_{i}$ is nonzero and $a_{1}>0$. Now it suffices to show that $K$ is not 2-bipolar.

Suppose $K$ is 2-bipolar. Then in [CK17, Subsection 2.3], a 1-negaton, denoted by $X^{-}$, with boundary $M\left(K_{1}\right)$ is constructed such that for

$$
P:=\operatorname{Ker}\left\{i_{*}: H_{1}\left(M\left(K_{1}\right) ; \mathbb{Q}\left[t^{ \pm 1}\right]\right) \longrightarrow H_{1}\left(X^{-} ; \mathbb{Q}\left[t^{ \pm 1}\right]\right)\right\}
$$

where $i_{*}$ is induced from the inclusion, either $P=\left\langle\alpha_{J}\right\rangle$ or $P=\left\langle\alpha_{D}\right\rangle$.

Case 1: $P=\left\langle\alpha_{D}\right\rangle$. In this case we follow the arguments in Cha19, Section 3]; the 4 -manifold $X^{-}$is modified to a new 4-manifold $X^{0}$, keeping the boundary, defined to be

$$
X^{0}:=V^{0} \cup_{\partial_{-} C} C \cup_{\partial_{+} C \backslash M\left(K_{1}\right)}\left(\left(a_{1}-1\right) Z_{1}^{0} \sqcup\left(\bigsqcup_{i>1}\left|a_{i}\right| Z_{i}^{0}\right) \sqcup Z_{L}^{0}\right) .
$$

See Figure 5. We refer the reader to Section 3 in Cha19 for the notations in the definition of $X^{0}$. 


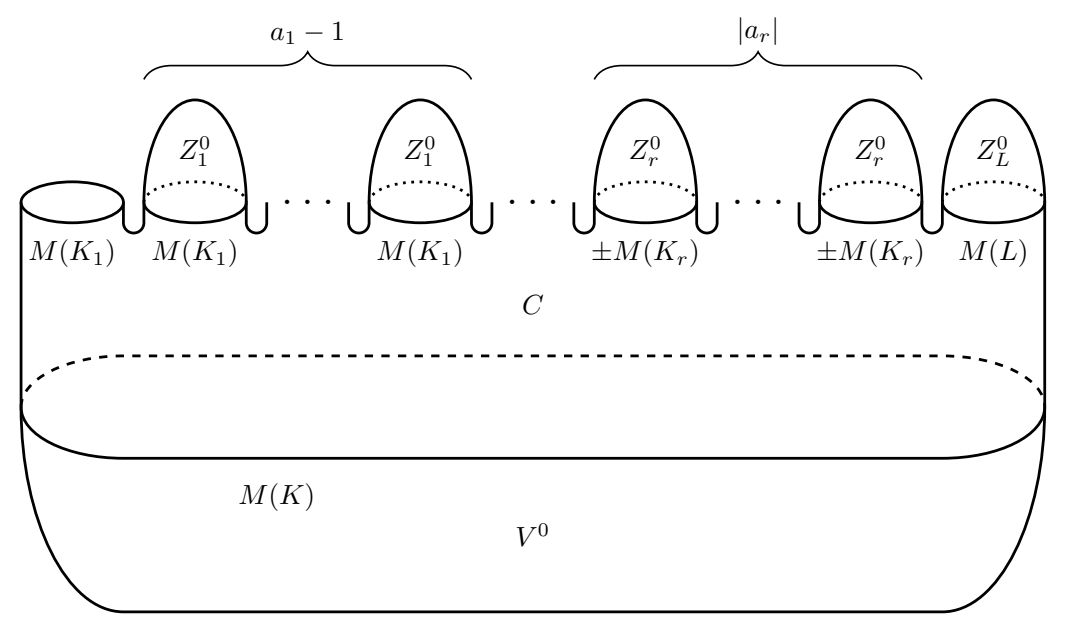

Figure 5. The 4-manifold $X^{0}$.

Remark 3.2. The above $X^{0}$ is not the same as $X=X_{0}$ defined in Cha19, Section 3] for the case $n=1$; it is the same as $X$ with $E_{0}$ removed, which is also the same as $X_{1}$ for $n=1$ in [Cha19, Section 3]. See Figure 5 in Cha19.

In Cha19, Section 3] it is shown that $\pi_{1}\left(X^{0}\right) \cong \pi_{1}\left(X^{-}\right)$and $H_{1}\left(X^{0} ; \mathbb{Q}\left[t^{ \pm 1}\right]\right) \cong$ $H_{1}\left(X^{-} ; \mathbb{Q}\left[t^{ \pm 1}\right]\right)$. It is also shown that

$$
P=\operatorname{Ker}\left\{i_{*}: H_{1}\left(M\left(K_{1}\right) ; \mathbb{Q}\left[t^{ \pm 1}\right]\right) \longrightarrow H_{1}\left(X^{0} ; \mathbb{Q}\left[t^{ \pm 1}\right]\right)\right\},
$$

which is $\left\langle\alpha_{D}\right\rangle$ by our hypothesis.

We will use $X^{0}$ and its subspaces to compute the von Neumann $\rho$-invariant of $M\left(K_{1}\right)$ defined in [CG85, and for more details of the von Neumann $\rho$-invariant we refer the reader to Cha19, Subsection 3.2]. We will use the same notations as in Cha19; suppose $M$ is a closed oriented 3-manifold and $W$ is a 4-manifold with $\partial W=M$. Suppose for a countable discrete group $\Gamma$ there is a homomorphism $\phi: \pi_{1}(M) \rightarrow \Gamma$ which extends to $\pi_{1}(W)$. Then, the von Neumann $\rho$-invariant of $M$ associated to $\phi$ is

$$
\rho(M, \phi)=\bar{\sigma}_{\Gamma}^{(2)}(W):=\operatorname{sign}_{\Gamma}^{(2)}(W)-\operatorname{sign}(W) .
$$

For our proof, we choose a group $\Gamma$ and a representation $\phi: \pi_{1}(X) \rightarrow \Gamma$ following Definition 3.1 in Cha19, wherein we take $n=1$ and replace $\mathbb{Z}_{p_{1}}$ by $\mathbb{Q}$. This is one of our key modifications for the proof of [Cha19, Theorem D]. More details follow: let

$$
\Sigma:=\left\{f(t) \in \mathbb{Q}\left[t^{ \pm 1}\right] \mid f(1) \neq 0, \operatorname{gcd}\left(f(t), \lambda_{m}\right)=1\right\} .
$$

Let $\pi:=\pi_{1}\left(X^{0}\right)$ and $\mathcal{P}^{1} \pi:=\pi^{(1)}=[\pi, \pi]$. Let $\mathcal{P}^{2} \pi$ be the kernel of the composition

$$
\begin{aligned}
\mathcal{P}^{1} \pi \longrightarrow \mathcal{P}^{1} \pi /\left[\mathcal{P}^{1} \pi, \mathcal{P}^{1} \pi\right] \otimes_{\mathbb{Z}} \mathbb{Q}=H_{1}\left(\pi ; \mathbb{Q}\left[t^{ \pm 1}\right]\right) \longrightarrow H_{1}\left(\pi ; \mathbb{Q}\left[t^{ \pm 1}\right] \Sigma^{-1}\right) \\
\longrightarrow H_{1}\left(X ; \mathbb{Q}\left[t^{ \pm 1}\right] \Sigma^{-1}\right) \longrightarrow H_{1}\left(X ; \mathbb{Q}\left[t^{ \pm 1}\right] \Sigma^{-1}\right) / \operatorname{Im} H_{1}\left(Z_{L}^{0} ; \mathbb{Q}\left[t^{ \pm 1}\right] \Sigma^{-1}\right),
\end{aligned}
$$

where $\operatorname{Im} H_{1}\left(Z_{L}^{0} ; \mathbb{Q}\left[t^{ \pm 1}\right] \Sigma^{-1}\right)$ is the image of $H_{1}\left(Z_{L}^{0} ; \mathbb{Q}\left[t^{ \pm 1}\right] \Sigma^{-1}\right)$ under the homomorphism induced from the inclusion from $Z_{L}^{0}$ to $X^{0}$.

Now we let $\Gamma:=\pi / \mathcal{P}^{2} \pi$ and let $\phi: \pi_{1}\left(X^{0}\right)=\pi \rightarrow \Gamma$ be the quotient map. By abuse of notation, we denote by $\phi$ the restrictions of $\phi$ to subspaces of $X^{0}$. 
By Novikov additivity, we have the following equation (compare it with Equation (3.2) in Cha19]).

$$
\rho\left(M\left(K_{1}\right), \phi\right)=\bar{\sigma}_{\Gamma}^{(2)}\left(V^{0}\right)+\bar{\sigma}_{\Gamma}^{(2)}(C)+\sum_{j=1}^{a_{1}-1} \bar{\sigma}_{\Gamma}^{(2)}\left(Z_{1, j}^{0}\right)+\sum_{i=2}^{r} \sum_{j=1}^{\left|a_{i}\right|} \bar{\sigma}_{\Gamma}^{(2)}\left(Z_{i, j}^{0}\right)+\bar{\sigma}_{\Gamma}^{(2)}\left(Z_{L}^{0}\right)
$$

where $Z_{i, j}^{0}$ are copies of $Z_{i}^{0}$. We compute each term of the above equation, and this will lead us to a contradiction.

(1) $\rho\left(M\left(K_{1}\right), \phi\right)=\int_{S^{1}} \sigma_{J_{1}}(\omega) d \omega$. To show this, we construct an integral 1-solution $Z$ for $M\left(K_{1}\right)$ following the arguments in COT04 (and also Cha13). The knot $R(U, D)$ where $U$ denotes the unknot is a slice knot whose slice disk is obtained by cutting the band dual to $\alpha_{D}$. Let $W$ be the exterior of the slice disk in the 4-ball, and hence $\partial W=R(U, D)$. Since $J_{1}$ is 0 -negative, it is also integrally 0 -solvable by CHH13, Proposition 5.5]. Let $W^{\prime}$ be an integral 0-solution with $\partial W^{\prime}=M\left(J_{1}\right)$. By doing surgery along the curves generating $\pi_{1}\left(W^{\prime}\right)^{(1)}$ we may assume that $\pi_{1}\left(W^{\prime}\right) \cong \mathbb{Z}$. Consider $M\left(J_{1}\right)$ as $\left(S^{3} \backslash \nu\left(J_{1}\right)\right) \cup$ $\left(S^{1} \times D^{2}\right)$ where $\nu\left(J_{1}\right)$ denotes the open tubular neighborhood of $J_{1}$. Now let $Z$ be the 4-manifold obtained from $W$ and $W^{\prime}$ by identifying the tubular neighborhood of the curve $\alpha_{J}$ in $M(R(U, D))=\partial W$ and $S^{1} \times D^{2} \subset M\left(J_{1}\right)=\partial W^{\prime}$. Then $Z$ is an integral 1-solution with $\partial Z=M\left(K_{1}\right)$.

Let

$$
\begin{aligned}
\Gamma^{\prime} & :=\left(H_{1}\left(X^{0} ; \mathbb{Q}\left[t^{ \pm 1}\right] \Sigma^{-1}\right) / \operatorname{Im} H_{1}\left(Z_{L}^{0} ; \mathbb{Q}\left[t^{ \pm 1}\right] \Sigma^{-1}\right)\right) \rtimes \mathbb{Z} \\
& \cong H_{1}\left(X^{0} \backslash Z_{L}^{0} ; \mathbb{Q}\left[t^{ \pm 1}\right] \Sigma^{-1}\right) \rtimes \mathbb{Z}
\end{aligned}
$$

where the last isomorphism is given by [Cha19, Subsection 3.1]. Here, in the semidirect products $\mathbb{Z}=H_{1}\left(X^{0}\right)$ acts on the left summands via deck transformations. Due to the definition of $\Gamma$, there is an injective homomorphism $i: \Gamma \hookrightarrow \Gamma^{\prime}$.

We assert that the composition $i \circ \phi: \pi_{1}\left(M\left(K_{1}\right)\right) \rightarrow \Gamma \hookrightarrow \Gamma^{\prime}$ extends to $\pi_{1}(Z)$. Recall that

$$
\left\langle\alpha_{D}\right\rangle=\operatorname{Ker}\left\{H_{1}\left(M\left(K_{1}\right) ; \mathbb{Q}\left[t^{ \pm 1}\right]\right) \longrightarrow H_{1}\left(X^{0} ; \mathbb{Q}\left[t^{ \pm 1}\right]\right)\right\} .
$$

Due to the definition of $\Sigma$ and the fact that $\Delta_{L}$ is coprime to $\Delta_{K_{1}}$, using Mayer-Vietoris sequences one can easily see that

$$
\left\langle\alpha_{D}\right\rangle=\operatorname{Ker}\left\{H_{1}\left(M\left(K_{1}\right) ; \mathbb{Q}\left[t^{ \pm 1}\right]\right) \longrightarrow H_{1}\left(X^{0} \backslash Z_{L}^{0} ; \mathbb{Q}\left[t^{ \pm 1}\right] \Sigma^{-1}\right)\right\} .
$$

Therefore, the map $i \circ \phi: \pi_{1}\left(M\left(K_{1}\right)\right) \rightarrow \Gamma \hookrightarrow \Gamma^{\prime}$ factors through the injective map

$$
\bar{\phi}:\left(H_{1}\left(M\left(K_{1}\right) ; \mathbb{Q}\left[t^{ \pm 1}\right]\right) /\left\langle\alpha_{D}\right\rangle\right) \rtimes \mathbb{Z} \longrightarrow \Gamma^{\prime} .
$$

Due to the construction of $W$, one can easily see that

$$
H_{1}\left(W ; \mathbb{Q}\left[t^{ \pm 1}\right]\right) \cong H_{1}\left(R ; \mathbb{Q}\left[t^{ \pm 1}\right]\right) /\left\langle\alpha_{D}\right\rangle \cong H_{1}\left(M\left(K_{1}\right) ; \mathbb{Q}\left[t^{ \pm 1}\right]\right) /\left\langle\alpha_{D}\right\rangle .
$$

Using Mayer-Vietoris sequences, one can see that $H_{1}\left(Z ; \mathbb{Q}\left[t^{ \pm 1}\right]\right) \cong H_{1}\left(W ; \mathbb{Q}\left[t^{ \pm 1}\right]\right)$, and therefore $H_{1}\left(Z ; \mathbb{Q}\left[t^{ \pm 1}\right]\right) \cong H_{1}\left(M\left(K_{1}\right) ; \mathbb{Q}\left[t^{ \pm 1}\right]\right) /\left\langle\alpha_{D}\right\rangle$. It follows that the map defined to be the composition

$$
\begin{aligned}
\pi_{1}(Z) \longrightarrow \pi_{1}(Z) / \pi_{1}(Z)^{(2)} \cong H_{1}\left(Z ; \mathbb{Z}\left[t^{ \pm 1}\right]\right) \rtimes \mathbb{Z} \\
\longrightarrow H_{1}\left(Z ; \mathbb{Q}\left[t^{ \pm 1}\right]\right) \rtimes \mathbb{Z} \cong\left(H_{1}\left(M\left(K_{1}\right) ; \mathbb{Q}\left[t^{ \pm 1}\right]\right) /\left\langle\alpha_{D}\right\rangle\right) \rtimes \mathbb{Z} \stackrel{\bar{\phi}}{\longrightarrow} \Gamma^{\prime}
\end{aligned}
$$

is an extension of $i \circ \phi: \pi_{1}\left(M\left(K_{1}\right)\right) \rightarrow \Gamma^{\prime}$, which is what we asserted. By abuse of notation we also denote by $\bar{\phi}$ the extension to $\pi_{1}(Z)$ and its restrictions to subspaces of $Z$.

By the subgroup property of the $\rho$-invariant (see Property (2.3) in [COT04, p. 108]), $\rho\left(M\left(K_{1}\right), \phi\right)=\rho\left(M\left(K_{1}\right), i \circ \phi\right)$. Therefore, we have $\rho\left(M\left(K_{1}\right), \phi\right)=\bar{\sigma}_{\Gamma^{\prime}}^{(2)}(Z)$, where 
$\bar{\sigma}_{\Gamma^{\prime}}^{(2)}(Z)=\bar{\sigma}_{\Gamma^{\prime}}^{(2)}(W)+\bar{\sigma}_{\Gamma^{\prime}}^{(2)}\left(W^{\prime}\right)$ by Novikov additivity. Since $W$ is a slice disk exterior, $\bar{\sigma}_{\Gamma^{\prime}}^{(2)}(W)=0$ by COT03, Theorem 4.2]. Since the curve $\alpha_{J}$ is of infinite order in $H_{1}\left(M\left(K_{1}\right) ; \mathbb{Q}\left[t^{ \pm 1}\right]\right) /\left\langle\alpha_{D}\right\rangle$, the subgroup of $\Gamma^{\prime}$ generated by $\bar{\phi}\left(\alpha_{J}\right)$ is isomorphic to $\mathbb{Z}$. Let $\mu_{J_{1}}$ be a meridian of $J_{1}$. By the identification of $W$ and $W^{\prime}$, we have $\bar{\phi}\left(\mu_{J_{1}}\right)=\bar{\phi}\left(\alpha_{J}\right)$. Since $\bar{\phi}$ on $\pi_{1}\left(M\left(J_{1}\right)\right)$ factors through $\pi_{1}\left(W^{\prime}\right) \cong \mathbb{Z}$ and $\bar{\phi}\left(\mu_{J_{1}}\right)$ is of infinite order in $\Gamma^{\prime}$, the image of $\pi_{1}\left(M\left(J_{1}\right)\right)$ under $\bar{\phi}$ is isomorphic to $\mathbb{Z}$. Therefore by [COT03, Proposition 5.13] and [COT04, Lemma 5.3], we obtain $\bar{\sigma}_{\Gamma^{\prime}}^{(2)}\left(W^{\prime}\right)=\rho\left(M\left(J_{1}\right), \bar{\phi}\right)=\int_{S^{1}} \sigma_{J_{1}}(\omega) d \omega$.

(2) $\bar{\sigma}_{\Gamma}^{(2)}\left(V^{0}\right)=\bar{\sigma}_{\Gamma}^{(2)}(C)=0$. This is proved in [Cha19, Subsection 3.2].

(3) $\bar{\sigma}_{\Gamma}^{(2)}\left(Z_{1, j}^{0}\right)=0$ or $-\int_{S^{1}} \sigma_{J_{i}}(\omega) d \omega$, and for $i \geq 2, \bar{\sigma}_{\Gamma}^{(2)}\left(Z_{i, j}^{0}\right)=0$ or $\pm \int_{S^{1}} \sigma_{J_{i}}(\omega) d \omega$. This follows from [CK17, Lemma 3.3]. (Lemma 3.3 in [CK17] uses $\mathbb{Z}_{p}$ coefficients, and its proof works with $\mathbb{Z}$ coefficients as well.) Or, one can also prove it using arguments similar to the one in (1) above.

(4) $\bar{\sigma}_{\Gamma}^{(2)}\left(Z_{L}^{0}\right)=0$. This is a key ingredient of the proof of [Cha19, Theorem D], and it is proved in [Cha19, Subsection 3.2]. For the reader's conveience, and since we use $\mathbb{Q}$ coefficients in place of $\mathbb{Z}_{p}$ coefficients which was used in Cha19, we give a brief sketch of the computation. Due to the definition of $\mathcal{P}^{2} \pi_{1}\left(X^{0}\right)$, the map $\phi$ on $\pi_{1}\left(Z_{L}^{0}\right)$ maps $\pi_{1}\left(Z_{L}^{0}\right)^{(1)}$ trivially into $\Gamma$. Therefore, the map $\phi$ on $\pi_{1}\left(Z_{L}^{0}\right)$ factors through the injective map $H_{1}\left(Z_{L}^{0}\right) \rightarrow \Gamma$, and therefore $\bar{\sigma}_{\Gamma}^{(2)}\left(Z_{L}^{0}\right)=\int_{S^{1}} \sigma_{L}(\omega) d \omega$ by COT03, Proposition 5.13] and [COT04, Lemma 5.3]. Since $L$ is concordant to the connected sum of $K$ and $-a_{i} K_{i}$ and each of $K$ and $K_{i}$ is 1-bipolar, $L$ is also 1-bipolar. Since $L$ is 1bipolar, it is algebraically slice by [CHH13, Corollary 5.7], and hence $\int_{S^{1}} \sigma_{L}(\omega) d \omega=0$.

From Equation (3.1) and the above computations (1)-(4), we conclude that for some $\epsilon_{i} \in \mathbb{Z}$ where $\epsilon_{1}>0$,

$$
\sum_{i=1}^{r}\left(\epsilon_{i} \cdot \int_{S^{1}} \sigma_{J_{i}}(\omega) d \omega\right)=0
$$

But it contradicts that $\int_{S^{1}} \sigma_{J_{i}}(\omega) d \omega$ are linearly independent over $\mathbb{Z}$ (see Lemma 3.1 (2)).

Case 2: $P=\left\langle\alpha_{J}\right\rangle$. In this case the proof is exactly the same as the proof for Case $P=\left\langle\alpha_{J}\right\rangle$ of Theorem D in Cha19, Section 4] except that we use Theorem 2.7, which is our key technical contribution in this case: let $K_{0}:=R(U, D)$. Let $\Sigma_{r}\left(K_{1}\right)$ (resp. $\left.\Sigma_{r}\left(K_{0}\right)\right)$ be the $r$-fold cyclic cover of $S^{3}$ branched along $K_{1}$ (resp. $K_{0}$ ). In Cha19 Section 4] it was asserted that $d\left(\Sigma_{r}\left(K_{0}\right), \mathfrak{s}_{\Sigma_{r}}+k \cdot \widehat{x_{1}}\right) \geq 0$ for all sufficiently large prime $r$ and for all $k \in \mathbb{Z}$ (see Lemma 4.1 in Cha19 and the paragraphs preceding Lemma 4.1) due to the fact that $J_{n-1}^{1}$ is unknotted by changing some positive crossings to negative crossings.

Note that our $J_{1}$ cannot be unknotted by changing positive crossings to negative crossings. But since $J_{1}$ is $\nu^{+}$-equivalent to the unknot (see Lemma $3.1(3)$ ), by Theorem 2.7

$$
d\left(\Sigma_{r}\left(K_{1}\right), \mathfrak{s}_{\Sigma_{r}}+k \cdot \widehat{x_{1}}\right)=d\left(\Sigma_{r}\left(K_{0}\right), \mathfrak{s}_{\Sigma_{r}}+k \cdot \widehat{x_{1}}\right)
$$

for all prime $r$ and for all $k \in \mathbb{Z}$. Since $M\left(K_{1}\right)$ bounds a 1-negaton $X^{-}$and

$$
\left\langle\alpha_{J}\right\rangle=\operatorname{Ker}\left\{H_{1}\left(M\left(K_{0}\right) ; \mathbb{Q}\left[t^{ \pm 1}\right]\right) \longrightarrow H_{1}\left(W ; \mathbb{Q}\left[t^{ \pm 1}\right]\right)\right\},
$$

using the same arguments as in the proof of [Cha19, Lemma 4.1], we obtain

$$
d\left(\Sigma_{r}\left(K_{1}\right), \mathfrak{s}_{\Sigma_{r}}+k \cdot \widehat{x_{1}}\right) \geq 0
$$

for all $k \in \mathbb{Z}$ and for all sufficiently large prime $r$. Now by Equation 3.2 above, we obtain the desired conclusion of Lemma 4.1 in Cha19. The rest of the proof for this 
case is exactly identical with the proof given in [Cha19, Section 4], and this completes the sketch of the proof of Theorem 1.2 .

\section{Appendix: metabolizers for Blanchfield forms and concordance invariants}

In Appendix, using metabelian concordance invariants given via metabolizers of the Blanchfield form of a knot, we give an obstruction for a knot $K$ to being concordant to any knot with Alexander polynomial coprime to $\Delta_{K}$ (see Theorem A.1). This obstruction is given by combining the results in [Kim05, KK08, Bao15, KK18.

We review the invariants and notations which will be used in Theorem A.1 below. Recall that $\mathbb{Z}=\langle t\rangle$ acts on $\mathbb{Q}(t) / \mathbb{Q}\left[t^{ \pm 1}\right]$ via multiplication. This action induces a semidirect product $\Gamma:=\left(\mathbb{Q}(t) / \mathbb{Q}\left[t^{ \pm 1}\right]\right) \rtimes \mathbb{Z}$. For a knot $K$, let $B \ell_{\mathbb{Q}}$ be the rational Blanchfield form

$$
B \ell_{\mathbb{Q}}: H_{1}\left(M(K) ; \mathbb{Q}\left[t^{ \pm 1}\right]\right) \times H_{1}\left(M(K) ; \mathbb{Q}\left[t^{ \pm 1}\right]\right) \longrightarrow \mathbb{Q}(t) / \mathbb{Q}\left[t^{ \pm 1}\right] .
$$

A choice of $x \in H_{1}\left(M(K) ; \mathbb{Q}\left[t^{ \pm 1}\right]\right)$ induces a homomorphism $\phi_{x}: \pi_{1}(M(K)) \rightarrow \Gamma$ defined by $\phi_{x}(y)=\left(B \ell_{\mathbb{Q}}\left(x, y \mu^{-\epsilon(y)}\right), \epsilon(y)\right)$ where $\left.\epsilon: \pi_{1}\left(M\left(K_{1}\right)\right)\right) \rightarrow \mathbb{Z}$ is the abelianization and $\mu$ is a meridian of $K$. Then, one can obtain the von Neumann $\rho$-invariant $\rho\left(M(K), \phi_{x}\right)$, whose definition can be found in Section 3 (The homomorphism $\phi_{x}$ was introduced in COT03, wherein our $\Gamma$ is denote by $\Gamma_{1}$ or $\Gamma_{1}^{U}$ and called the rationally universal 1-solvable group.)

For a rational homology 3-sphere $Y$ and a $\operatorname{Spin}^{c}$ structure $\mathfrak{s}$ on $Y$, we let

$$
\bar{d}(Y, \mathfrak{s}):=d(Y, \mathfrak{s})-d\left(Y, \mathfrak{s}_{0}\right)
$$

where $d$ is the correction term invariant of Ozsváth and Szabó OS03a and $\mathfrak{s}_{0}$ denotes the canonical $\operatorname{Spin}^{c}$ structure on $Y$. Let $n$ be a prime power and $\zeta_{n}$ be the primitive $n$th root of unity. For $\Sigma_{r}(K)$, the $r$-fold cyclic cover of $S^{3}$ branched along a knot $K$, and a character of prime power order $\chi: H_{1}\left(\Sigma_{r}(K)\right) \rightarrow \mathbb{Q} / \mathbb{Z}$, we denote by $\tau(K, \chi) \in$ $L_{0}\left(\mathbb{Q}\left(\zeta_{n}\right)\right) \otimes_{\mathbb{Z}} \mathbb{Q}$ the Casson-Gordon invariant associated to $\chi$ CG86.

Let

$$
B \ell_{\mathbb{Z}}: H_{1}\left(M(K) ; \mathbb{Z}\left[t^{ \pm 1}\right]\right) \times H_{1}\left(M(K) ; \mathbb{Z}\left[t^{ \pm 1}\right]\right) \longrightarrow S^{-1} \mathbb{Z}\left[t^{ \pm 1}\right] / \mathbb{Z}\left[t^{ \pm 1}\right]
$$

be the Blanchfield form where $S:=\left\{f(t) \in \mathbb{Z}\left[t^{ \pm 1}\right] \mid f(1)=1\right\}$. For a $\mathbb{Z}\left[t^{ \pm 1}\right]$-module $M$ and $r \geq 1$, the map $\pi^{r}: M \rightarrow M /\left(t^{r}-1\right) M$ is the quotient map.

Theorem A.1. Let $K$ and $J$ be knots with coprime Alexander polynomials. Suppose $K \# J$ is slice. Then, there exists a $\mathbb{Z}\left[t^{ \pm 1}\right]$-submodule $P_{K}$ of $H_{1}\left(M(K) ; \mathbb{Z}\left[t^{ \pm 1}\right]\right)$ which is a metabolizer with respect to the Blanchfield form $B \ell_{\mathbb{Z}}$ for $K$ such that all of the following hold:

(1) $P_{K} \otimes_{\mathbb{Z}} \mathbb{Q}$ is a metabolizer with respect to the rational Blanchfield form $B \ell_{\mathbb{Q}}$ for $K$ and $\rho\left(M(K), \phi_{x}\right)=0$ for all $x \in P_{K} \otimes_{\mathbb{Z}} \mathbb{Q}$.

(2) There exists a set of finitely many primes $S=\left\{p_{1}, p_{2}, \ldots, p_{m}\right\}$ such that if $r=p^{k}$ is a prime power where $p \notin S$, then $\pi^{r}\left(P_{K}\right)$ is a metabolizer with respect to the linking form $H_{1}\left(\Sigma_{r}(K)\right) \times H_{1}\left(\Sigma_{r}(K)\right) \rightarrow \mathbb{Q} / \mathbb{Z}$ and the following hold:

(a) $\bar{d}\left(\Sigma_{r}(K), \mathfrak{s}_{\mathrm{o}}+\widehat{c}\right)=0$ for all $c \in \pi^{r}\left(P_{K}\right)$, and

(b) for every character of prime power order $\chi: H_{1}\left(\Sigma_{r}(K)\right) \rightarrow \mathbb{Q} / \mathbb{Z}$ which vanishes on $\pi^{r}\left(P_{K}\right)$, the Casson-Gordon invariant $\tau(K, \chi)$ is constant. Moreover, if $K$ is algebraically slice, then $\tau(K, \chi)$ vanishes.

The proof of Theorem A.1 is postponed to the end of Appendix. The set of primes $S$ in Theorem A.1 can be taken as the empty set if $K \# J$ bounds a slice disk $D$ in the 
4-ball such that, letting $W$ denote the exterior of the slice disk $D, H_{1}\left(W ; \mathbb{Z}\left[t^{ \pm 1}\right]\right)$ has no $\mathbb{Z}$-torsion elements. In particular, if $K \# J$ is a ribbon knot, then $S$ is the empty set.

Proposition A.2. Let $R$ be the knot $9_{46}$, which is $R_{1}$ in Figure 1 . Then, the knot $R\left(D_{+}\left(T_{2,3}, 0\right) \#-T_{2,3}, D_{+}\left(T_{2,3}, 0\right)\right)$ is topologically slice and not concordant to any knot with Alexander polynomial coprime to $\lambda_{1}=(t-2)(2 t-1)$.

Proof. Let $J:=D_{+}\left(T_{2,3}, 0\right) \#-T_{2,3}$ and $D:=D_{+}\left(T_{2,3}, 0\right)$. Let $K:=R(J, D)$. The knot $K$ is the satellite knot of winding number 0 whose pattern knot is $R(J, U)$ and companion knot is $D$. Therefore, since $D$ is topologically slice, $K$ is topologically slice.

Suppose $K \# L$ is slice for a knot $L$ whose Alexander polynomial is coprime to $\lambda_{1}$. Then by Theorem A.1, there exist a $\mathbb{Z}\left[t^{ \pm 1}\right]$-submodule $P_{K}$ of $H_{1}\left(M(K) ; \mathbb{Z}\left[t^{ \pm 1}\right]\right)$, which is a metabolizer with respect to the Blanchfield form $B \ell_{\mathbb{Z}}$, and a set of finitely many primes $S$ which satisfies the conditions (1) and (2) in Theorem A.1.

For brevity, let $P:=P_{K}$. Let $\alpha_{J}$ and $\alpha_{D}$ be the curves depicted in Figure1 One can easily compute that

$$
H_{1}\left(R(J, D) ; \mathbb{Z}\left[t^{ \pm 1}\right]\right) \cong H_{1}\left(R ; \mathbb{Z}\left[t^{ \pm 1}\right]\right) \cong \mathbb{Z}\left[t^{ \pm 1}\right] /\langle t-2\rangle \oplus \mathbb{Z}\left[t^{ \pm 1}\right] /\langle 2 t-1\rangle
$$

where the summands are $\left\langle\alpha_{J}\right\rangle$ and $\left\langle\alpha_{D}\right\rangle$, which are the submodules generated by the curves $\alpha_{J}$ and $\alpha_{D}$, respectively. One can also see that $R(J, D)$ and $R$ have isomorphic Blanchfield forms, and $\left\langle\alpha_{J}\right\rangle$ and $\left\langle\alpha_{D}\right\rangle$ are the only metabolizers with respect the Blanchfield form on $H_{1}\left(M(K) ; \mathbb{Z}\left[t^{ \pm 1}\right]\right)$. Therefore the submodule $P$ is either $\left\langle\alpha_{J}\right\rangle$ or $\left\langle\alpha_{D}\right\rangle$.

Suppose $P=\left\langle\alpha_{J}\right\rangle$. In this case we use Theorem A.1 (2) (a); for an odd prime $r \notin S$, $\bar{d}\left(\Sigma_{r}(K), \mathfrak{s}_{0}+\widehat{c}\right)=0$ for all $c \in \pi^{r}(P)$. The subgroup $\pi^{r}(P)$ of $H_{1}\left(\Sigma_{r}(K)\right) \cong \mathbb{Z}_{2^{r}-1} \oplus$ $\mathbb{Z}_{2^{r}-1}$ is generated by a lift of $\alpha_{J}$ in $\Sigma_{r}(K)$, say $x$, and hence we have $\bar{d}\left(\Sigma_{r}(K), \mathfrak{s}_{0}+k \cdot \widehat{x}\right)=$ 0 for all $k \in \mathbb{Z}$. Recall from Remark 2.3 that $J$ is $\nu^{+}$-equivalent to the unknot. Since $\Sigma_{r}(K)$ is a $\mathbb{Z}_{2}$-homology sphere, by Theorem 2.7

$$
\bar{d}\left(\Sigma_{r}(K), \mathfrak{s}_{0}+k \cdot \widehat{x}\right)=\bar{d}\left(\Sigma_{r}(R(U, D)), \mathfrak{s}_{0}+k \cdot \widehat{x}\right)
$$

for all $k \in \mathbb{Z}$. Since $R(U, D)$ is slice, $d\left(\Sigma_{r}(R(U, D)), \mathfrak{s}_{0}\right)=0$, and hence

$$
d\left(\Sigma_{r}(R(U, D)), \mathfrak{s}_{0}+k \cdot \widehat{x}\right)=\bar{d}\left(\Sigma_{r}(R(U, D)), \mathfrak{s}_{0}+k \cdot x\right)=0
$$

for all $k \in \mathbb{Z}$. But by [CK17, Theorem 5.4],

$$
d\left(\Sigma_{r}(R(U, D)), \mathfrak{s}_{0}+2^{r-1} \cdot \widehat{x}\right) \leq-\frac{3}{2},
$$

which is a contradiction.

Suppose $P=\left\langle\alpha_{D}\right\rangle$. In this case, we use Theorem A.1 (1), which implies that $\rho\left(M(K), \phi_{x}\right)=0$ for all $x \in P \otimes_{\mathbb{Z}} \mathbb{Q}$. Choose a nonzero $x \in P \otimes_{\mathbb{Z}} \mathbb{Q}$, for instance, $x=\alpha_{D} \otimes 1$. Let $W$ be the exterior of the slice disk for $R(U, D)$ which is obtained by cutting the band dual to the curve $\alpha_{D}$ in Figure 1. Then $\partial W=R(U, D)$. Let $W^{\prime}$ be a 4-manifold with $\partial W^{\prime}=M(J)$ such that the inclusion induces an isomorphism $H_{1}(M(J)) \stackrel{\cong}{\rightarrow} H_{1}\left(W^{\prime}\right)$. The existence of $W^{\prime}$ follows from that the bordism group $\Omega_{3}\left(S^{1}\right)=0$. We can also arrange that $\pi_{1}\left(W^{\prime}\right) \cong \mathbb{Z}$ by doing surgeries along the curves generating $\pi_{1}\left(W^{\prime}\right)^{(1)}$. Note that $M(J)=\left(S^{3} \backslash \nu(J)\right) \cup\left(S^{1} \times D^{2}\right)$ where $\nu(J)$ is the open tubular neighborhood of $J$ in $S^{3}$. Let $X$ be the 4-manifold obtained from $W$ and $W^{\prime}$ by identifying the tubular neighborhood of $\alpha_{J}$ in $R(U, D)=\partial W$ and $S^{1} \times D^{2} \subset M(J)=\partial W^{\prime}$. Then $\partial X=M(K)$ and

$$
\operatorname{Ker}\left\{H_{1}\left(M(K) ; \mathbb{Q}\left[t^{ \pm 1}\right]\right)\right\} \longrightarrow H_{1}\left(X ; \mathbb{Q}\left[t^{ \pm 1}\right]\right)=P \otimes_{\mathbb{Z}} \mathbb{Q} .
$$

Since $\mathbb{Q}\left[t^{ \pm 1}\right]$ is a $\mathrm{PID}, x \in P \otimes_{\mathbb{Z}} \mathbb{Q}$, and $P \otimes_{\mathbb{Z}} \mathbb{Q}$ is a metabolizer with respect to the rational Blanchfield form on $H_{1}\left(M(K) ; \mathbb{Q}\left[t^{ \pm 1}\right]\right)$, by COT03, Theorem 3.6] the homomorphism $\phi_{x}$ extends to $\pi_{1}(X)$. 
Therefore, $\rho\left(M(K), \phi_{x}\right)=\bar{\sigma}_{\Gamma}^{(2)}(W)+\bar{\sigma}_{\Gamma}^{(2)}\left(W^{\prime}\right)$. Since $W$ is a slice disk exterior, $\bar{\sigma}_{\Gamma}^{(2)}(W)=0$ by COT03, Theorem 4.2]. Since $\partial W^{\prime}=M(J)$, we have $\bar{\sigma}_{\Gamma}^{(2)}\left(W^{\prime}\right)=$ $\rho\left(M(J), \phi_{x}\right)$ where by abuse of notation $\phi_{x}$ also denotes the restriction of $\phi_{x}$ to $\pi_{1}(M(J))$. Since $x$ is a nonzero element in $P=\left\langle\alpha_{D}\right\rangle$, we have $B \ell_{\mathbb{Q}}\left(x, \alpha_{J}\right) \neq 0$ for the rational Blanchfield form $B \ell_{\mathbb{Q}}$ on $H_{1}\left(M(K), \mathbb{Q}\left[t^{ \pm 1}\right]\right)$. Also note that $\epsilon\left(\alpha_{J}\right)=0$ where $\epsilon: \pi_{1}(M(K)) \rightarrow \mathbb{Z}$ is the abelianization. Since $\phi_{x}\left(\alpha_{J}\right)=\left(B \ell_{\mathbb{Q}}\left(x, \alpha_{J}\right), \epsilon\left(\alpha_{J}\right)\right)$, the subgroup of $\Gamma$ generated by $\phi_{x}\left(\alpha_{J}\right)$ is isomorphic to $\mathbb{Z}$. By our construction of $X$, the meridian, say $\mu$, of $J$ is identified with the curve $\alpha_{J}$ in $X$, and hence $\phi_{x}(\mu)$ is not trivial in $\Gamma$. Since $\phi_{x}$ on $\pi_{1}(M(J))$ factors through $\pi_{1}\left(W^{\prime}\right) \cong \mathbb{Z}$, the image of $\pi_{1}(M(J))$ in $\Gamma$ is also isomorphic to $\mathbb{Z}$. Now by [COT03, Proposition 5.13] and [COT04, Lemma 5.3], $\bar{\sigma}_{\Gamma}^{(2)}\left(W^{\prime}\right)=\rho\left(M(J), \phi_{x}\right)=\int_{S^{1}} \sigma_{J}(\omega) d \omega=\frac{2}{3}$. Therefore $\rho\left(M(K), \phi_{x}\right)=0+\frac{2}{3} \neq 0$, which is a contradiction.

We finish Appendix with the proof of Theorem A.1

Proof of Theorem A.1. Let $L:=K \# J$ and $W$ be the exterior of a slice disk for $L$ in the 4-ball. Let $F H_{1}\left(W ; \mathbb{Z}\left[t^{ \pm 1}\right]\right):=H_{1}\left(W ; \mathbb{Z}\left[t^{ \pm 1}\right]\right) / T$ where $T$ is the $\mathbb{Z}$-torsion submodule of $H_{1}\left(W ; \mathbb{Z}\left[t^{ \pm 1}\right]\right)$. Let

$$
P_{L}:=\operatorname{Ker}\left\{i: H_{1}\left(M(L) ; \mathbb{Z}\left[t^{ \pm 1}\right]\right) \stackrel{i_{\mathbb{Z}}}{\longrightarrow} H_{1}\left(W ; \mathbb{Z}\left[t^{ \pm 1}\right]\right) \stackrel{\pi}{\longrightarrow} F H_{1}\left(W ; \mathbb{Z}\left[t^{ \pm 1}\right]\right)\right\}
$$

where $i_{\mathbb{Z}}$ is the inclusion-induced homomorphism and $\pi$ is the quotient map. It is well known that $P_{L}$ is a metabolizer with respect to the the Blanchfield form $B \ell_{\mathbb{Z}}$ (see [Fri03, Proposition 2.7]).

Note that $H_{1}\left(M(L) ; \mathbb{Z}\left[t^{ \pm 1}\right]\right) \cong H_{1}\left(M(K) ; \mathbb{Z}\left[t^{ \pm 1}\right]\right) \oplus H_{1}\left(M(J) ; \mathbb{Z}\left[t^{ \pm 1}\right]\right)$. Now let

$$
P_{K}:=P_{L} \cap H_{1}\left(M(K) ; \mathbb{Z}\left[t^{ \pm 1}\right]\right)=\left\{x \in H_{1}\left(M(K) ; \mathbb{Z}\left[t^{ \pm 1}\right]\right) \mid(x, 0) \in P_{L}\right\} .
$$

Similarly, we let $P_{J}:=P_{L} \cap H_{1}\left(M(J) ; \mathbb{Z}\left[t^{ \pm 1}\right]\right)$. We will show that $P_{K}$ satisfies the desired properties. We need a lemma:

Lemma A.3. $P_{L} \cong P_{K} \oplus P_{J}$ as $\mathbb{Z}\left[t^{ \pm 1}\right]$-modules and $P_{K}$ and $P_{J}$ are metabolizers for the Blanchfield forms on $H_{1}\left(M(K) ; \mathbb{Z}\left[t^{ \pm 1}\right]\right)$ and $H_{1}\left(M(J) ; \mathbb{Z}\left[t^{ \pm 1}\right]\right)$, respectively.

Proof. It is obvious that $P_{K} \oplus P_{J} \subset P_{L}$. Let $(x, y) \in P_{L}$ where $x \in H_{1}\left(M(K) ; \mathbb{Z}\left[t^{ \pm 1}\right]\right)$ and $y \in H_{1}\left(M(J) ; \mathbb{Z}\left[t^{ \pm 1}\right]\right)$. We will show that $x \in P_{K}$ and $y \in P_{J}$, which will imply that $P_{L} \subset P_{K} \oplus P_{J}$.

Since $\mathbb{Q}\left[t^{ \pm 1}\right]$ is a PID and $\Delta_{K}$ and $\Delta_{J}$ are coprime, there exist $\bar{f}(t)$ and $\bar{g}(t)$ in $\mathbb{Q}\left[t^{ \pm 1}\right]$ such that $\bar{f}(t) \Delta_{K}+\bar{g}(t) \Delta_{J}=1$. Then, there exists some nonzero integer $c$ such that letting $f(t):=c \bar{f}(t)$ and $g(t):=c \bar{g}(t)$, we have $f(t) \Delta_{K}+g(t) \Delta_{J}=c$ and $f(t), g(t) \in$ $\mathbb{Z}\left[t^{ \pm 1}\right]$. Recall that $(x, y) \in P_{L}$. Since $\Delta_{K}$ and $\Delta_{J}$ annihilate $H_{1}\left(M(K) ; \mathbb{Z}\left[t^{ \pm 1}\right]\right)$ and $H_{1}\left(M(J) ; \mathbb{Z}\left[t^{ \pm 1}\right]\right)$, respectively, in $H_{1}\left(M(K) ; \mathbb{Z}\left[t^{ \pm 1}\right]\right)$ we have

$$
c x=\left(f(t) \Delta_{K}+g(t) \Delta_{J}\right) x=\left(g(t) \Delta_{J}\right) x .
$$

Also, in $H_{1}\left(M(J) ; \mathbb{Z}\left[t^{ \pm 1}\right]\right)$ we have $\left(g(t) \Delta_{J}\right) y=0$. Therefore, in $H_{1}\left(M(L) ; \mathbb{Z}\left[t^{ \pm 1}\right]\right)$ we have $g(t) \Delta_{J}(x, y)=(c x, 0)$, and hence $(c x, 0) \in P_{L}$.

Consider the following commutative diagram.

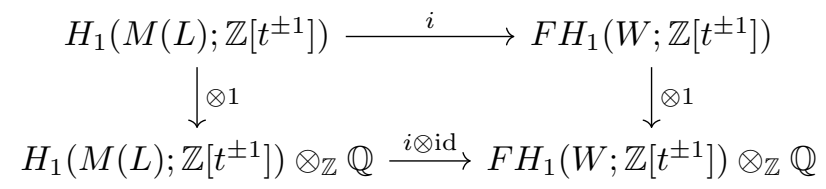

Since $(c x, 0) \in P_{L}=\operatorname{Ker}(i)$, it follows that $(i \otimes \mathrm{id})((c x) \otimes 1,0)=0$. Therefore

$$
(i \otimes \mathrm{id})(x \otimes 1,0)=\frac{1}{c} \cdot(i \otimes \mathrm{id})((c x) \otimes 1,0)=0,
$$


and hence $(x \otimes 1,0) \in \operatorname{Ker}(i \otimes \mathrm{id})$. Since the vertical maps of the above diagram are injective, we have $(x, 0) \in \operatorname{Ker}(i)=P_{L}$, and hence $x \in P_{K}$. Similarly, one can show that $y \in P_{J}$, and it follows that $P_{L} \subset P_{K} \oplus P_{J}$ and hence $P_{L} \cong P_{K} \oplus P_{J}$. The modules $P_{K}$ and $P_{L}$ are metabolizers due to [KK18, Lemma 3.1] and its proof.

One can see that $\rho\left(M(L), \phi_{z}\right)=0$ for all $z \in P_{L} \otimes_{\mathbb{Z}} \mathbb{Q}$ since

$$
P_{L} \otimes_{\mathbb{Z}} \mathbb{Q}=\operatorname{Ker}\left\{i_{\mathbb{Q}}: H_{1}\left(M(L) ; \mathbb{Q}\left[t^{ \pm 1}\right]\right) \longrightarrow H_{1}\left(W ; \mathbb{Q}\left[t^{ \pm 1}\right]\right)\right\}
$$

where $W$ is the exterior of a slice disk (see Theorems 3.6 and 4.2 in COT03). By Lemma A.3. $P_{L} \otimes_{\mathbb{Z}} \mathbb{Q} \cong\left(P_{K} \otimes_{\mathbb{Z}} \mathbb{Q}\right) \oplus\left(P_{J} \otimes_{\mathbb{Z}} \mathbb{Q}\right)$, and $P_{L} \otimes_{\mathbb{Z}} \mathbb{Q}, P_{K} \otimes_{\mathbb{Z}} \mathbb{Q}$, and $P_{J} \otimes_{\mathbb{Z}} \mathbb{Q}$ are metabolizers for the rational Blanchfield forms. Now by [KK08, Theorem 3.1] and its proof, $\rho\left(M(K), \phi_{x}\right)=0$ for all $x \in P_{K} \otimes_{\mathbb{Z}} \mathbb{Q}$. This shows the property (1).

We show the property $(2)$. Recall that $F H_{1}\left(W ; \mathbb{Z}\left[t^{ \pm 1}\right]\right)=H_{1}\left(W ; \mathbb{Z}\left[t^{ \pm 1}\right]\right) / T$ where $T$ is the $\mathbb{Z}$-torsion submodule. Since multiplication by $t-1$ induces an automorphism on $H_{1}\left(W ; \mathbb{Z}\left[t^{ \pm 1}\right]\right.$ ) (see Mil68), by Lev77, Lemma (3.1)], the subgroup $T$ is finite. Therefore, there are only finitely many primes $q_{1}, q_{2}, \ldots, q_{\ell}$ which divide the order of $T$. Then, by [Bao15, Lemma 2.3] there exists a set of finitely may primes $S=\left\{p_{1}, p_{2}, \ldots, p_{m}\right\}$ such that if $p$ is a prime not in $S$ and $r=p^{k}$ for some $k \geq 1$, then any $q_{i}$, which divides the order of $T$, does not divide the order of $H_{1}\left(\Sigma_{r}(K)\right)$.

Let $D$ be the slice disk for $L$ whose exterior is $W$. Denote the $r$-fold cyclic cover of the 4-ball branched along $D$ by $\Sigma_{r}(D)$. Then, it follows from Mil68] that

$$
\pi^{r}\left(H_{1}\left(M(L) ; \mathbb{Z}\left[t^{ \pm 1}\right]\right)\right) \cong H_{1}\left(\Sigma_{r}(L)\right) \text { and } \pi^{r}\left(H_{1}\left(W ; \mathbb{Z}\left[t^{ \pm 1}\right]\right)\right) \cong H_{1}\left(\Sigma_{r}(D)\right) .
$$

Now suppose $r=p^{k}$, a prime power, where $p \notin S$. We have the following commutative diagram where the homomorphisms $i_{\mathbb{Z}}$ and $j$ are induced from inclusions and $\pi$ is the quotient map.

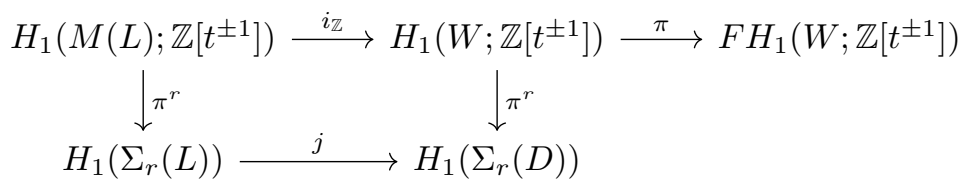

Restricting the right vertical map $\pi^{r}$ to $\operatorname{Im}\left(i_{\mathbb{Z}}\right)$, by our choice of $S$ and $r$, we obtain the following commutative diagram

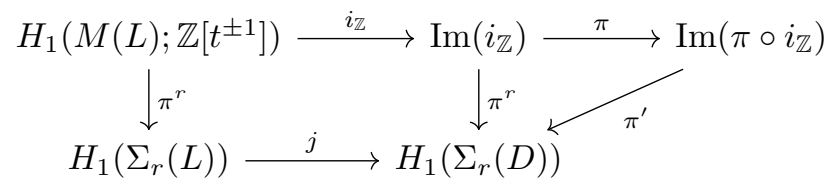

where $\pi^{\prime}$ is the quotient map. Now $\pi^{r}\left(P_{L}\right) \subset \operatorname{Ker}(j)$ since $P_{L}=\operatorname{Ker}\left(\pi \circ i_{\mathbb{Z}}\right)$. It is well known that $\operatorname{Ker}(j)$ is a metabolizer with respect to the linking form on $H_{1}\left(\Sigma_{r}(L)\right)$. Also, since $P_{L}$ is a metabolizer for the Blanchfield form on $H_{1}\left(M(L) ; \mathbb{Z}\left[t^{ \pm 1}\right]\right)$, the subgroup $\pi^{r}\left(P_{L}\right)$ of $H_{1}\left(\Sigma_{r}(L)\right)$ is a metabolizer for the linking form (see [Fri03, Proposition 2.18]). Since $\pi^{r}\left(P_{L}\right) \subset \operatorname{Ker}(j)$ and $\left|\pi^{r}\left(P_{L}\right)\right|^{2}=|\operatorname{Ker}(j)|^{2}=\left|H_{1}\left(\Sigma_{r}(L)\right)\right|$, it follows that $\pi^{r}\left(P_{L}\right)=\operatorname{Ker}(j)$.

Let $c \in \pi^{r}\left(P_{K}\right)$. We follow the arguments in Bao15 to show the property (2)(a). Note that $\pi^{r}\left(P_{L}\right)=\pi^{r}\left(P_{K}\right) \oplus \pi^{r}\left(P_{J}\right)$, and hence $(c, 0) \in \pi^{r}\left(P_{L}\right)$. Since $\pi^{r}\left(P_{L}\right)=\operatorname{Ker}(j)$ where $\Sigma_{r}(D)$ is the cyclic cover of the 4 -ball branched along the slice disk $D$, it follows that $d\left(\Sigma_{r}(L), \mathfrak{s}_{\mathcal{O}}+\widehat{(c, 0)}\right)=0$. By the additivity of the $d$-invariant, we have

$$
0=d\left(\Sigma_{r}(L), \mathfrak{s}_{0}+\widehat{(c, 0)}\right)=d\left(\Sigma_{r}(K), \mathfrak{s}_{0}+\widehat{c}\right)+d\left(\Sigma_{r}(J), \mathfrak{s}_{0}\right),
$$


which holds for any choice of $c \in \pi^{r}\left(P_{K}\right)$. Therefore, for each $c \in \pi^{r}\left(P_{K}\right)$,

$$
\begin{aligned}
\bar{d}\left(\Sigma_{r}(K), \mathfrak{s}_{0}+\widehat{c}\right) & =d\left(\Sigma_{r}(K), \mathfrak{s}_{0}+\widehat{c}\right)-d\left(\Sigma_{r}(K), \mathfrak{s}_{0}\right) \\
& =-d\left(\Sigma_{r}(J), \mathfrak{s}_{0}\right)-\left(-d\left(\Sigma_{r}(J), \mathfrak{s}_{0}\right)\right. \\
& =0,
\end{aligned}
$$

which shows the property (2)(a).

To show the property (2)(b), we follow the arguments in $\operatorname{Kim05}$. Let $\chi: H_{1}\left(\Sigma_{r}(K)\right) \rightarrow$ $\mathbb{Q} / \mathbb{Z}$ be a character of prime power order which vanishes on $\pi^{r}\left(P_{K}\right)$. Then the character

$$
\chi \oplus 0: H_{1}\left(\Sigma_{r}(L)\right) \cong H_{1}\left(\Sigma_{r}(K)\right) \oplus H_{1}\left(\Sigma_{r}(J)\right) \longrightarrow \mathbb{Q} / \mathbb{Z}
$$

vanishes on $\pi^{r}\left(P_{K}\right) \oplus \pi^{r}\left(P_{J}\right)=\pi^{r}\left(P_{L}\right)$. Therefore, since $\pi^{r}\left(P_{L}\right)=\operatorname{Ker}(j)$, it follows that $\tau(L, \chi \oplus 0)=0$. Now by the additivity of the Casson-Gordon invariant, we have $0=\tau(L, \chi \oplus 0)=\tau(K, \chi)+\tau(J, 0)$. Therefore, $\tau(K, \chi)=-\tau(J, 0)$, a constant. If $K$ is algebraically slice, since $K \# J$ is slice by the hypothesis, $J$ is also algebraically slice, and it follows that $\tau(J, 0)=0$.

\section{References}

[AK80] Selman Akbulut and Robion Kirby, Branched covers of surfaces in 4-manifolds, Math. Ann 252 (1979/80), no. 2, 111-131.

[Bao15] Yuanyuan Bao, Polynomial splittings of Ozsváth and Szabó's d-invariant, Topology Proc. 46 (2015), 309-322.

[CG85] Jeff Cheeger and Mikhail Gromov, Bounds on the von Neumann dimension of $L^{2}$-cohomology and the Gauss-Bonnet theorem for open manifolds, J. Differential Geom. 21 (1985), no. 1, $1-34$.

[CG86] Andrew Casson and Cameron Gordon, Cobordism of classical knots, À la recherche de la topologie perdue, Birkhäuser Boston, Boston, MA, 1986, With an appendix by Patrick M. Gilmer, pp. 181-199.

[CH15] Tim D. Cochran and Peter Horn, Structure in the bipolar filtration of topologically slice knots, Algebr. Geom. Topol. 15 (2015), no. 1, 415-428.

[Cha13] Jae Choon Cha, Amenable $L^{2}$-theoretic methods and knot concordance, Int. Math. Res. Not. IMRN (2013), no. 15, 1-36.

[Cha19] Jae Choon Cha, Primary decomposition in the concordance group of topologically slice knots arXiv:1910.14629, 2019.

[CHH13] Tim D. Cochran, Shelly Harvey, and Peter Horn, Filtering smooth concordance classes of topologically slice knots, Geom. Topol. 17 (2013), no. 4, 2103-2162.

[CK17] Jae Choon Cha and Min Hoon Kim, The bipolar filtration of topologically slice knots, arXiv preprint arXiv:1710.07803, 2017.

[COT03] Tim D. Cochran, Kent E. Orr, and Peter Teichner, Knot concordance, Whitney towers and $L^{2}$-signatures, Ann. of Math. (2) 157 (2003), no. 2, 433-519.

[COT04] Tim D. Cochran, Kent E. Orr, and Peter Teichner, Structure in the classical knot concordance group, Comment. Math. Helv. 79 (2004), no. 1, 105-123.

[DHSL19] Irving Dai, Jennifer Hom, Matthew Stoffregen, and Truong Linh, More concordance homomorphisms from knot floer homology, arXiv:1902.03333, 2019

[Don83] Simon K. Donaldson, An application of gauge theory to four-dimensional topology, J. Differential Geom. 18 (1983), no. 2, 279-315.

[End95] Hisaki Endo, Linear independence of topologically slice knots in the smooth cobordism group, Topology Appl. 63 (1995), no. 3, 257-262.

[FQ90] Michael H. Freedman and Frank Quinn, Topology of 4-manifolds, Princeton Mathematical Series, vol. 39, Princeton University Press, Princeton, NJ, 1990.

[Fre82a] Michael H. Freedman, A surgery sequence in dimension four; the relations with knot concordance, Invent. Math. 68 (1982), no. 2, 195-226.

[Fre82b] Michael H. Freedman, The topology of four-dimensional manifolds, J. Differential Geom. 17 (1982), no. 3, 357-453.

[Fre84] Michael H. Freedman, The disk theorem for four-dimensional manifolds, Proceedings of the International Congress of Mathematicians, Vol. 1, 2 (Warsaw, 1983) (Warsaw), PWN, 1984 pp. $647-663$ 
[Fri03] Stefan Friedl, Eta invariants as sliceness obstructions and their relation to Casson-Gordon invariants, ProQuest LLC, Ann Arbor, MI, 2003, Thesis (Ph.D.)-Brandeis University.

[GJ11] Joshua Greene and Stanislav Jabuka, The slice-ribbon conjecture for 3-stranded pretzel knots, Amer. J. Math. 133 (2011), no. 3, 555-580.

[GL92] Patrick M. Gilmer and Charles Livingston, The Casson-Gordon invariant and link concordance, Topology 31 (1992), no. 3, 475-492.

[GRS08] J. Elisenda Grigsby, Daniel Ruberman, and Sašo Strle, Knot concordance and Heegaard Floer homology invariants in branched covers, Geom. Topol. 12 (2008), no. 4, 2249-2275.

[Hed07] Matthew Hedden, Knot Floer homology of Whitehead doubles, Geom. Topol. 11 (2007), no. 4, $2277-2338$

[HK11] Matthew Hedden and Paul Kirk, Chern-Simons invariants, SO(3) instantons, and $\mathbb{Z} / 2$ homology cobordism, Chern-Simons gauge theory: 20 years after, AMS/IP Stud. Adv. Math., vol. 50, Amer. Math. Soc., Providence, RI, 2011, pp. 83-114.

[HK12] Matthew Hedden and Paul Kirk, Instantons, concordance, and Whitehead doubling, J. Differential Geom. 91 (2012), no. 2, 281-319.

[HKL16] Matthew Hedden, Se-Goo Kim, and Charles Livingston, Topologically slice knots of smooth concordance order two, J. Differential Geom. 102 (2016), no. 3, 353-393.

[HLR12] Matthew Hedden, Charles Livingston, and Daniel Ruberman, Topologically slice knots with nontrivial Alexander polynomial, Adv. Math. 231 (2012), no. 2, 913-939.

[Hom14] Jennifer Hom, The knot Floer complex and the smooth concordance group, Comment. Math. Helv. 89 (2014), no. 3, 537-570.

[Hom15] Jennifer Hom, On the concordance genus of topologically slice knots, Int. Math. Res. Not. IMRN (2015), no. 5, 1295-1314.

[Hom17] Jennifer Hom, A survey on Heegaard Floer homology and concordance, J. Knot Theory Ramifications 26 (2017), no. 2, 1740015.

[HW16] Jennifer Hom and Zhongtao Wu, Four-ball genus bounds and a refinement of the OzváthSzabó tau invariant, J. Symplectic Geom. 14 (2016), no. 1, 305-323.

[Jab12] Stanislav Jabuka, Concordance invariants from higher order covers, Topology Appl. 159 (2012), no. 10-11, 2694-2710.

[JN07] Stanislav Jabuka and Swatee Naik, Order in the concordance group and Heegaard Floer homology, Geom. Topol. 11 (2007), 979-994.

[Kim05] Se-Goo Kim, Polynomial splittings of Casson-Gordon invariants, Math. Proc. Cambridge Philos. Soc. 138 (2005), no. 1, 59-78.

[KK08] Se-Goo Kim and Taehee Kim, Polynomial splittings of metabelian von Neumann rhoinvariants of knots, Proc. Amer. Math. Soc. 136 (2008), no. 11, 4079-4087.

[KK18] Se-Goo Kim and Taehee Kim, Polynomial splittings of correction terms and doubly slice knots, J. Knot Theory Ramifications 27 (2018), no. 1, 1850001, 9.

[KP18] Min Hoon Kim and Kyungbae Park, An infinite-rank summand of knots with trivial Alexander polynomial, J. Symplectic Geom. 16 (2018), no. 6, 1749-1771.

[Lev77] Jerome P. Levine, Knot modules. I, Trans. Amer. Math. Soc. 229 (1977), 1-50.

[LR14] Adam S. Levine and Daniel Ruberman, Generalized Heegaard Floer correction terms, Proceedings of the Gökova Geometry-Topology Conference 2013, Gökova Geometry/Topology Conference (GGT), Gökova, 2014, pp. 76-96.

[Mil68] John W. Milnor, Infinite cyclic coverings, Conference on the Topology of Manifolds (Michigan State Univ., E. Lansing, Mich., 1967), Prindle, Weber \& Schmidt, Boston, Mass., 1968, pp. 115-133.

[MO07] Ciprian Manolescu and Brendan Owens, A concordance invariant from the Floer homology of double branched covers, Int. Math. Res. Not. IMRN (2007), no. 20, Art. ID rnm077, 21.

[NW15] Yi Ni and Zhongtao Wu, Cosmetic surgeries on knots in $S^{3}$, J. Reine Angew. Math. 706 (2015), 1-17.

[OS03a] Peter Ozsváth and Zoltán Szabó, Absolutely graded Floer homologies and intersection forms for four-manifolds with boundary, Adv. Math. 173 (2003), no. 2, 179-261.

[OS03b] Peter Ozsváth and Zoltán Szabó, Knot Floer homology and the four-ball genus, Geom. Topol. 7 (2003), 615-639 (electronic).

[OSS17] Peter Ozsváth, András I. Stipsicz, and Zoltán Szabó, Concordance homomorphisms from knot Floer homology, Adv. Math. 315 (2017), 366-426.

[Sat18] Kouki Sato, A full-twist inequality for the $\nu^{+}$-invariant, Topology Appl. 245 (2018), 113-130.

[Sat19] Kouki Sato, The $\nu^{+}$-equivalence classes of genus one knots, arXiv:1907.09116, 2019. 
Department of Mathematics, POSTECH, Pohang Gyeongbuk 37673, Republic of Korea

E-mail address: kminhoon@gmail.com

Department of Mathematics and Research Institute for Basic Sciences, Kyung Hee University, Seoul 02447, Republic of Korea

E-mail address: sgkim@khu.ac.kr

Department of Mathematics, Konkuk University, Seoul 05029, Republic of Korea

E-mail address: tkim@konkuk.ac.kr 